TRANSACTIONS OF THE

AMERICAN MATHEMATICAL SOCIETY

Volume 296, Number 2, August 1986

\title{
ON THE COVERING DIMENSION OF THE SET OF SOLUTIONS OF SOME NONLINEAR EQUATIONS
}

\author{
P. M. FITZPATRICK, I. MASSABÓ AND J. PEJSACHOWICZ
}

\begin{abstract}
We prove an abstract theorem whose sole hypothesis is that the degree of a certain map is nonzero and whose conclusions imply sharp, multidimensional continuation results. Applications are given to nonlinear partial differential equations.
\end{abstract}

1. Introduction and statements of the main results. Let $X$ be a Banach space, $m$ be a positive integer, and $O$ be an open subset of $\mathbf{R}^{m} \times X$. Suppose that $f: \bar{O} \rightarrow X$ is continuous. It is our objective here to present rather precise results on the connectivity and the covering dimension of certain subsets of the set of solutions of the equation

$$
f(v)=0, \quad v \in \overline{0}
$$

Since equation (1.1) is underdetermined, at least in principle, it seems natural that under not overly restrictive assumptions one should be able to not only guarantee the existence of a solution of equation (1.1), but one should also be able to describe the size of the set of solutions in terms of the integer $m$. We specifically remark that we make no assertion concerning the existence of some distinguished set of trivial solutions of equation (1.1), so this is not a bifurcation problem.

For a general topological space, $V$, there is the classical notion of covering dimension, $\operatorname{dim}(V)$, and, speaking generally, our conclusions are of the type where we give lower bounds on the covering dimension at each point of certain connected subsets of $\{v \in \bar{O} \mid f(v)=0\}$. In the case when $f$ is a $C^{1}$ mapping, of which 0 is a regular value, it is known that one can give a rather precise description of the local structure of the solutions of equation (1.1). In contradistinction, both our assumptions and conclusions are global and do not involve smoothness assumptions.

Certain classes of linear elliptic partial differential operators may be expressed as operators $\mathcal{L}: Z \rightarrow Y$, where $Z$ and $Y$ are Banach spaces, and $\mathcal{L}$ is an operator which is Fredholm of index $m$, where $m$ is a positive integer. If $F: Z \rightarrow Y$ is a nonlinear compact operator and one considers the equation

$$
\mathcal{L}(z)+F(z)=0, \quad z \in \bar{U},
$$

where $U$ is an open subset of $Z$, then equation (1.2) may be reformulated in the form of equation (1.1).

Moreover, it is clear that many problems explicitly depending on an $m$-dimensional parameter can be written in the form (1.1).

Received by the editors September 1, 1982.

1980 Mathematics Subject Classification. Primary 35J65, 47H09, 47H17.

(c) 1986 American Mathematical Society $0002-9947 / 86 \$ 1.00+\$ .25$ per page 
To state our results we need to introduce the idea of a complementing mapping. Suppose that $f(\lambda, x)=x-C(\lambda, x)$ for $\lambda \in \mathbf{R}^{m}$ and $x \in X$ with $(\lambda, x) \in \overline{0}$, where $C$ is a compact mapping in the sense that $C$ is continuous and $C(D)$ has compact closure when $D \subseteq \bar{O}$ is bounded. We will say that a bounded, continuous mapping $g: \bar{O} \rightarrow \mathbf{R}^{m}$ is a complement for $f: \bar{O} \rightarrow X$ provided that the mapping $h: \overline{0} \subseteq \mathbf{R}^{m} \times X \rightarrow \mathbf{R}^{m} \times X$, defined by $h(\lambda, x)=(g(\lambda, x), f(\lambda, x))$ for $(\lambda, x) \in \overline{0}$, has nonzero Leray-Schauder degree. Recall that a map is called bounded when it maps bounded sets into bounded sets. Observe that $h$ is a compact perturbation of the identity. Also, since we have not assumed 0 to be bounded, our hypothesis that the Leray-Schauder degree of $h$ on $\overline{0}$ is nonzero means that $h^{-1}(0) \cap \bar{\sigma}$ is a compact subset of $O$ and $\operatorname{deg}(h, U, 0) \neq 0$, when $U$ is any bounded neighborhood of $h^{-1}(0)$.

In the case when $f$ is linear it can be complemented precisely when it is surjective and has an $m$-dimensional kernel. More generally, when $f$ is differentiable, $f\left(\lambda_{0}, x_{0}\right)=0$ and $\partial f\left(\lambda_{0}, x_{0}\right) / \partial x$ is a bijection on $X$, the usual proof of the implicit function theorem uses the idea of a complementing map to reduce the proof to an application of the inverse function theorem.

There are two quite general situations under which $f$ can be comples.ented. First, if $\left(\lambda_{0}, x_{0}\right) \in \mathcal{O}, O_{\lambda_{0}}=\left\{x \in X \mid\left(\lambda_{0}, x\right) \in O\right\}$, and $f_{\lambda_{0}}$ is the restriction of $f$ to $\left\{\lambda_{0}\right\} \times O_{\lambda_{0}}$, then $f$ is complemented by $g$, where $g(\lambda, x)=\lambda-\lambda_{0}$, provided that $\operatorname{deg}\left(f_{\lambda_{0}}, O_{\lambda_{0}}, 0\right) \neq 0$. Secondly, assuming $X=\mathbf{R}^{k}, O$ is bounded, $\partial O$ is smooth, $f$ is smooth, and 0 is a regular value both of $f: 0 \rightarrow \mathbf{R}^{k}$ and of $f: \partial O \rightarrow \mathbf{R}^{k}$, then $f$ can be complemented if and only if $f^{-1}(0) \cap \partial 0 \neq \varnothing$. The former result is a useful way of obtaining a complement. The latter result essentially states that if the linearization of $f$ can be complemented at each point (i.e., 0 is a regular value), then $f$ itself can be complemented under the necessary and sufficient condition that $f^{-1}(0) \cap \partial O \neq \varnothing$. These results are proven in $\S 2$.

Recall that if $V$ is any topological space and $n$ is a nonnegative integer, then the covering dimension of $V, \operatorname{dim}(V)$, equals $n$ provided that $n$ is the smallest integer with the property that whenever $\xi$ is an open cover of $V$ there is a refinement of $\mathcal{F}, \mathcal{F}^{\prime}$, such that $\mathcal{F}^{\prime}$ also covers $V$ and no more than any $n+1$ member of $\mathcal{F}^{\prime}$ have nonempty intersection. In this paper, $V$ is always a separable metric space, so that the above definition of dimension coincides with the notion of inductive dimension (see [10]). If $v_{0} \in V$, we will say that $\operatorname{dim}(V) \geq j$ at $v_{0}$ if each neighborhood of $v_{0}$ has dimension at least $j$.

Our two basic results are the following.

THEOREM 1.1. Let $X$ be a Banach space, $m$ be a positive integer and $O \subseteq$ $\mathbf{R}^{m} \times X$ be open. Assume $f: \overline{0} \rightarrow X$ is complemented by the mapping $g: \overline{0} \rightarrow \mathbf{R}^{m}$. Then there is a connected subset, $C$, of $f^{-1}(0)$ whose dimension at each point in $C \cap \mathcal{O}$ is at least $m$, which intersects $g^{-1}(0)$, and, moreover, has at least one of the following two properties:

(i) $C$ is unbounded.

(ii) $\operatorname{dim}(C \cap \partial O) \geq m-1$ and $g: C \cap \partial O \rightarrow \mathbf{R}^{m}-\{0\}$ is essential; in particular, when $m=1, C \cap \partial 0$ has at least two points.

Recall that if $A$ is a locally compact topological space, then a proper mapping $h: A \rightarrow \mathbf{R}^{m}$ is called essential among proper mappings provided that the natural 
continuous extension of $h$ from the one-point compactification of $A$ to $S^{m}$ is not homotopic to a constant map.

THEOREM 1.2. Suppose that the assumptions of Theorem 1.1 hold. In addition, suppose that $\operatorname{dim}\left(f^{-1}(0) \cap \partial 0\right)<m-1$ and that $g: f^{-1}(0) \rightarrow \mathbf{R}^{m}$ is proper. Then there exists a connected component, $C$, of $f^{-1}(0)$, whose dimension at each point is at least $m$ and such that $g: C \rightarrow \mathbf{R}^{m}$ is essential among proper mappings. In particular, $g(C)=\mathbf{R}^{m}$.

In the literature, the antecedents of these precise dimension conclusions on the set of solutions of a nonlinear equation are quite few. In [20], Rabinowitz considered an equation of the form (1.2), and under the assumption that $F$ is odd and $U=B(0, r)$ he gave a lower bound on the genus of $\{z \in Z \mid\|z\|=r, \mathcal{L}(z)+F(z)=0\}$. Using the relation between genus and dimension for symmetric subsets of $\partial B(0, R)$, the conclusion of $[\mathbf{2 0}]$ amounts to the assertion that $\operatorname{dim}\{z \mid z \in \partial U, \mathcal{L}(z)+F(z)=0\} \geq$ $m-1$. The methods of [20] are firmly rooted in the oddness assumption on $F$. On the other hand, we have not been able to deduce the most general form of the results of [20] from those presented here.

In [16], the second and third named authors obtained the following corollary of Theorem 1.2 under the additional assumption that $f^{-1}(0) \cap \partial 0=\varnothing$.

COROllary 1.1. Let $X$ be a Banach space, $m$ be a positive integer and $0 \subseteq$ $\mathbf{R}^{m} \times X$ be open. Let $C: \overline{0} \rightarrow X$ be compact and define $f: \bar{O} \rightarrow X$ by $f(\lambda, x)=$ $x-C(\lambda, x)$ for $(\lambda, x) \in \bar{O}$. Suppose $\lambda_{0} \in \mathbf{R}^{m}$ is such that $\operatorname{deg}\left(f_{\lambda_{0}}, O_{\lambda_{0}}, 0\right) \neq 0$. Finally assume $\operatorname{dim}\left(f^{-1}(0) \cap \partial 0\right)<m-1$ and that whenever $\left\{\lambda_{n}\right\} \subseteq \mathbf{R}^{m}$ is bounded and $\left\{\left(\lambda_{n}, x_{n}\right)\right\} \subseteq \overline{\mathcal{O}}$ is such that $\left\{f\left(\lambda_{n}, x_{n}\right)\right\}$ is bounded, then $\left\{x_{n}\right\}$ is bounded. Then the conclusion of Theorem 1.2 follows.

While the literature on results yielding assertions on the covering dimension of subsets of the set of solutions of equation (1.1) is somewhat sparce, there is an ample literature yielding connectivity properties of such subsets. The basic such result is the following: Let $U$ be an open bounded subset of a Banach space $X$, and let $H:[0,1] \times \bar{U} \rightarrow X$ be a compact perturbation of the projection onto $X$; if $H(t, x) \neq 0$ when $t \in[0,1], x \in \partial U$, and $\operatorname{deg}(H(0, \cdot), U, 0) \neq 0$, then there is a connected subset of $[0,1] \times U$ on which $H$ vanishes and which intersects both $\{0\} \times U$ and $\{1\} \times U$. The origin of this type of result may be found in [15], while the first precise formulation may be found in [5]. The connectivity assertion of Corollary 1.1 may be regarded as an extension of this result. We would like to emphasize that unlike various connectivity results where one assumes $f^{-1}(0)$ does not intersect certain subsets of the domain of $f$, we impose a restriction on the size of $f^{-1}(0) \cap \partial 0$. As far as we know, even connectivity results under such a weak assumption are new.

Actually, we are able to prove somewhat stronger results than we have stated above. When $X$ and $Y$ are Banach spaces with certain approximation properties, there is a wide class of mappings, $h: \bar{O} \subseteq X \rightarrow Y$, the approximation-proper mappings, for which Browder and Petryshyn $[8]$ have defined a topological degree. When $X=Y$, and $X$ has a Schauder basis, this class includes compact perturbations of the identity. Our results hold in the context of approximation-proper mappings. $\S 3$ is devoted to a precise formulation of these more general results. While the proofs 
of the theorems for this class of mappings require little extra work, the more general formulation allows applications to broader classes of differential equations.

$\S 4$ is devoted to the proofs of our main results. In barest outline, our assumptions in terms of topological degree are translated into a statement of the action of a mapping in homology; this, in turn, yields a conclusion in cohomology; finally, we use the cohomological characterisation of dimension to obtain our conclusions. The crucial part of the proof is the case when $X=\mathbf{R}^{k}$, while the general case follows by approximation. We note that in [2] Alexander and Yorke used global cohomological methods to obtain a generalization of the implicit function theorem.

In $\S 5$ we consider two examples of the types of partial differential equations whose sets of solutions may be described by Theorems 1.1 and 1.2. The first is a nonlinear eigenvalue problem

$$
\begin{aligned}
& \sum_{|\alpha| \leq k}(-1)^{|\alpha|} D^{\alpha}\left(A_{\alpha}\left(x, \xi_{k}(u)(x)\right)\right) \\
& \quad=\sum_{|\beta| \leq k-1}(-1)^{|\beta|} D^{\beta}\left(B_{\beta}\left(\lambda, x, \xi_{k-1}(u)(x)\right)\right), \quad x \in \Omega .
\end{aligned}
$$

Here $\Omega$ is a bounded open subset of $\mathbf{R}^{n}, \xi_{s}(u)$ denotes the $s$-jet of $u$ for $u \in$ $W^{2 k, p}(\Omega), \lambda \in \mathbf{R}^{m}$, and $A_{\alpha}$ and $B_{\beta}$ are nonlinear functions of their appropriate arguments. Letting $V$ be a closed subspace of $W^{2 k, p}(\Omega)$ which contains $W_{0}^{2 k, p}(\Omega)$, we seek variational solutions of (1.3) which lie in $V$. By imposing the Leray-Lions growth and sign conditions on the $A_{\alpha}$ 's (see [14]) and quite general growth conditions on the $B_{\beta}$ 's, we are able to use an argument of Browder [7] to formulate equation (1.3) as an $m$-parameter approximation-proper mapping. Under additional mild assumptions on the $B_{\beta}$ 's we can conclude that there exists a connected subset, $C$, in $\mathbf{R}^{m} \times V$, of variational solutions of equation (1.3), whose dimension is at least $m$ at each point, and whose projection onto $\mathbf{R}^{m}$ covers $\mathbf{R}^{m}$. The second problem which we consider in $\S 5$ is

$$
\left\{\begin{array}{l}
\sum_{|\alpha| \leq 2 k} a_{\alpha}(x) D^{\alpha}(u)(x)=f\left(x, \xi_{2 k}(u)(x)\right), \quad x \in \Omega \\
B_{i}(u)(x)=0, \quad x \in \partial \Omega, 1 \leq i \leq k-1 .
\end{array}\right.
$$

The linear operator on the left-hand side is assumed to be uniformly elliptic, and we also assume that the boundary operators cover this elliptic operator [1]. In this case the left-hand side gives rise to a linear Fredholm operator, acting between suitable Sobolev spaces. We suppose the index of this operator is equal to $m \geq$ 0 . Under the assumption that $f$ has sublinear growth and satisfies asymptotic conditions at $\infty$ similar to those imposed for the well-investigated case when $m=0$, we are again able to give a precise dimensional and connectivity description of solutions of equation (1.4). Our results include those of [3 and 16], in the sense that our hypotheses are weaker and, in the case of $[\mathbf{3}]$, the conclusions are sharper. We observe that certain connectivity conclusions, without assertions concerning dimension, have recently been obtained for equation (1.4) in [18].

When the right-hand side of (1.4) depends on $2 k$ th derivatives of $u$ we need to use the generalized version of the theorems described in $\S 3$ : when the dependence is on lower order derivatives we can apply the results stated in the introduction. 
We wish to mention that connectivity result, but not dimension results, for certain classes of nonlinear equations have recently been obtained by Furi and Pera [12], where these authors adjoin a mapping to their original mapping and impose the assumption that the product of these two mappings be o-regular.

ACKNOWLEDGEMENTS. This work was completed under the auspices of the C.N.R. The first named author was also partially supported by an N.S.F. Grant.

2. In this section we will prove some results on the notion of complementing mapping. We refer the reader to Dold [9] for the definition and development of certain results on fundamental classes which we will need in this and the succeeding sections.

Recall that when $M$ is an oriented $k$-manifold without boundary and $\varphi: M \rightarrow \mathbf{R}^{k}$ is continuous and such that $\varphi^{-1}(0)$ is compact, then the degree of $\varphi$ on $M$ with respect to 0 , which we denote by $\operatorname{deg}(\varphi, M, 0)$, is defined by the formula

$$
\varphi_{*}\left(O_{\varphi^{-1}(0)}\right)=\operatorname{deg}(\varphi, M, 0) \cdot O_{0} \text {. }
$$

Here $\varphi_{*}: H_{k}\left(M, M-\varphi^{-1}(0)\right) \rightarrow H_{k}\left(\mathbf{R}^{k}, \mathbf{R}^{k}-0\right)$ is the mapping induced by $\varphi$ in homology, $O_{\varphi^{-1}(0)}$ is the fundamental class of $M$ around $\varphi^{-1}(0)$, and $O_{0}$ is the fundamental class of $\mathbf{R}^{k}$ around $\{0\}$ (see [9, Chapter 8]). The above definition yields a degree which has all of the useful properties associated with the case when $M$ is an open subset of $\mathbf{R}^{k}$, namely, the classical Brouwer degree.

Recall, also, that if $M$ is a differentiable $k$-manifold and $\varphi: M \rightarrow \mathbf{R}^{j}$ is differentiable, then 0 is called a regular value of $\varphi$ provided that $d \varphi(x)$ has maximal rank when $x \in M$ with $\varphi(x)=0$. In this case $\varphi^{-1}(0)$ is a $k-j$ dimensional oriented manifold.

PROPOSITION 2.1. Suppose $O \subseteq \mathbf{R}^{n+m}$ is open and $f: \bar{O} \rightarrow \mathbf{R}^{n}$ is continuous. Let $g: \bar{O} \rightarrow \mathbf{R}^{m}$ be smooth with 0 a regular value for $g: 0 \rightarrow \mathbf{R}^{m}$. Moreover, suppose $\{x \mid x \in \bar{O} ; f(x)=0, g(x)=0\}$ is a compact subset of 0 . Then, if $F: \bar{O} \rightarrow \mathbf{R}^{n+m}$ is defined by $F=(g \times f) \circ \Delta$, where $\Delta$ is the diagonal mapping, it follows that

$$
\operatorname{deg}(F, O, 0)=\operatorname{deg}(f, M, 0),
$$

where $M \equiv\{x \mid x \in \mathcal{O}, g(x)=0\}$ is oriented with the orientation induced by $g$.

PROOF. By invoking the tubular neighborhood theorem, we may choose a neighborhood, $W$, of $M$ in 0 , and a neighborhood, $W^{\prime}$, of $\{0\} \times M$ in $\mathbf{R}^{m} \times M$, together with a diffeomorphism $\phi: W \rightarrow W^{\prime}$ such that the following diagram commutes:

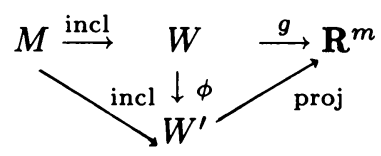

If we equip $M$ with the orientation induced by $g$, then $\phi$ becomes orientation preserving, so that from the composition property of degree,

$$
\operatorname{deg}(F, O, 0)=\operatorname{deg}\left(F \circ \phi^{-1}, W^{\prime}, 0\right) .
$$

Let us define $H:[0,1] \times W^{\prime} \rightarrow \mathbf{R}^{m} \times \mathbf{R}^{n}$ by $H(t,(x, y))=\left(x,\left(f \circ \phi^{-1}\right)(t x, y)\right)$. The commutativity of the above diagram implies $\{(x, y) \mid H(t,(x, y))=0$ for some $\left.t \in[0,1],(x, y) \in W^{\prime}\right\} \subset\{0\} \times M$. Hence the homotopy is admissible, so that 
$\operatorname{deg}\left(H(0, \cdot), W^{\prime}, 0\right)=\operatorname{deg}\left(H(1, \cdot), W^{\prime}, 0\right)$. The above diagram also implies $H(1, \cdot)=$ $F \circ \phi^{-1}$. On the other hand, $H(0,(x, y))=\left(x,\left(f \circ \phi^{-1}\right)(0, y)\right)=(x, f(y))$. Thus from the product property of degree

$$
\operatorname{deg}(F, O, 0)=\operatorname{deg}(f, M, 0) .
$$

Corollary 2.1. Let $f$ and $g$ be as in Proposition 2.1. Then $f$ is complemented by $g$ if and only if $\operatorname{deg}(f, M, 0) \neq 0$.

Now the simplest mapping which has 0 as a regular value is a projection mapping. Hence we obtain the following.

COROLlaRY 2.2. Let $0 \subseteq \mathbf{R}^{n+m}$ be open, and let $f: \bar{\emptyset} \rightarrow \mathbf{R}^{n}$ be continuous. Let $y_{0} \in \mathbf{R}^{m}$, and let $O_{y_{0}}=\left\{x \in \mathbf{R}^{n} \mid\left(x, y_{0}\right) \in \mathcal{O}\right\}$. Letting $f_{y_{0}}$ be the restriction of $f$ to $O_{y_{0}}$, assume $\operatorname{deg}\left(f_{y_{0}}, O_{y_{0}}, 0\right) \neq 0$. Then $f$ is complemented by $\pi-y_{0}$, where $\pi$ is the projection of $\mathbf{R}^{n+m}$ onto $\mathbf{R}^{m}$.

REMARK 2.1. An examination of the proof of Proposition 2.1 in the case when $g(\lambda, x)=\lambda-\lambda_{0}$ for $(\lambda, x) \in O \subseteq \mathbf{R}^{m} \times \mathbf{R}^{n}$ reveals that in this case the proof also carries over to the case when $f: \overline{0} \in \mathbf{R}^{m} \times X \rightarrow X, X$ being a Banach space, and $f(\lambda, x)=x-C(\lambda, x)$, where $C$ is compact. Thus such an $f$ can be complemented by $g(\lambda, x)=\lambda-\lambda_{0}$ precisely when $\operatorname{deg}\left(f_{\lambda_{0}}, O_{\lambda_{0}}, 0\right) \neq 0$, where now deg denotes the Leray-Schauder degree.

REMARK 2.2. Whenever $f: \bar{O} \rightarrow \mathbf{R}^{n}$ can be complemented, it can be complemented by a $g$ satisfying the assumptions of Proposition 2.1. This follows immediately from Sard's Theorem and the invariance of degree under small perturbations.

It is clear that a linear map of $\mathbf{R}^{n+m}$ to $\mathbf{R}^{n}$ can be complemented precisely when it is surjective, so that if $M$ is an $(n+m)$-manifold and $f: M \rightarrow \mathbf{R}^{n}$ then to say $d f(x)$ can be complemented when $x \in f^{-1}(0)$ is simply the assumption that 0 is a regular value for $f$. We will now give a necessary and sufficient condition for $f$ to have a complement, under the assumption that its linearization can be complemented. To this end we first recall some useful concepts.

Closely allied to the above definition of degree is the concept of winding number. Namely, if $M$ is a compact, orientable, $(k-1)$-dimensional manifold, and $\varphi: M \rightarrow$ $\mathbf{R}^{k}-0$, the winding number of $\varphi$ on $M$, denoted by $\mathrm{w}(\varphi, M)$, is defined by

$$
\varphi_{*}\left(O_{M}\right)=\mathrm{w}(\varphi, M) e,
$$

where $O_{M}$ is the fundamental class of $M$ and $e$ is the image of $O_{0}$ under the boundary map $\partial: H_{k}\left(\mathbf{R}^{k}, \mathbf{R}^{k}-0\right) \rightarrow H_{k-1}\left(\mathbf{R}^{k}-0\right)$.

From this definition, together with the definition of degree and some well-known relations between orientation of a manifold with boundary and the orientation of the boundary, one readily obtains the following result. (See the argument used in the proof of Proposition 4.9 of Dold [9, Chapter 8], and also, using somewhat different notation, that of Lemma 7 of Spanier [22, §3, Chapter 6].

PROPOSITION 2.2. Let $M$ be an oriented, compact, $k$-manifold with boundary. Let $f: M \rightarrow \mathbf{R}^{k}$ be continuous with $f(\partial M) \subseteq \mathbf{R}^{k}-0$. Then, if $\stackrel{\circ}{M}=M \backslash \partial M$

$$
\operatorname{deg}(f, \stackrel{\circ}{M}, 0)=\mathrm{w}(f, \partial M) \text {. }
$$


PROPOSITION 2.3. If $M$ is a compact, oriented ( $k-1)$-manifold, then there exists $g: M \rightarrow \mathbf{R}^{k}-0$ such that $\mathrm{w}(g, M) \neq 0$.

This is a direct consequence of the Hopf Classification Theorem, [22], since the winding number of $g$ on $M$ is the sum of the winding numbers of $g$ on each connected component of $M$.

THEOREM 2.1. Let $O \subseteq \mathbf{R}^{n+m}$ be open and bounded. Suppose $\partial 0$ is a smooth $(n+m-1)$-manifold and $f: \bar{O} \rightarrow \mathbf{R}^{n}$ is such that 0 is a regular value both of $f: 0 \rightarrow \mathbf{R}^{n}$ and of $f: \partial \mathcal{O} \rightarrow \mathbf{R}^{n}$. Moreover, assume $f^{-1}(0) \cap \partial \mathcal{0} \neq \varnothing$. Then $f: \bar{O} \rightarrow \mathbf{R}^{n}$ can be complemented.

ProOF. Let $M=f^{-1}(0)$. Then $\partial M=M \cap \partial O$ and $\partial M$ is a compact, oriented, $(m-1)$-manifold. By virtue of Proposition 2.3 we may choose $g: \partial M \rightarrow S^{m-1} \subseteq$ $\mathbf{R}^{m}$ such that $\mathrm{w}(g, \partial M) \neq 0$ in $H_{m-1}\left(\mathbf{R}^{m}-0\right)$. Extend $g$ to all of 0 .

From Proposition 2.2 we know $\operatorname{deg}(g, \stackrel{\circ}{M}, 0) \neq 0$. On the other hand if we invoke Proposition 2.1 with the roles of $f$ and $g$ interchanged we obtain

$$
\operatorname{deg}(F, O, 0)=\operatorname{deg}(g, \stackrel{\circ}{M}, 0),
$$

where $F=(g \times f) \circ \Delta$.

Thus $\operatorname{deg}(F, O, 0) \neq 0$; so $g$ complements $f$.

REMARK 2.3. In order for $f$ to have a complement it is also necessary that $f^{-1}(0) \cap \partial O \neq \varnothing$; this is an immediate consequence of Theorem 1.1. Moreover, simple examples show that in the above theorem one cannot drop the assumption that $\partial O$ is smooth.

REMARK 2.4. A satisfactory extension of Theorem 2.1 to an infinite-dimensional setting requires that one leave the context of the degree theories considered in this paper and instead consider the degree defined for nonlinear Fredholm mappings. We consider this question in [11].

3. Our purpose here is to describe a generalization of Theorems 1.1 and 1.2 to a situation when $f$ has compatible Galerkin approximations.

DEFINITION 3.1. Let $E$ and $W$ be Banach spaces. A triple of sequences $\Gamma=$ $\left\langle\left\{E_{n}\right\},\left\{W_{n}\right\},\left\{Q_{n}\right\}\right\rangle$ will be called an approximation scheme for mappings from $E$ to $W$ provided that $\left\{E_{n}\right\}$ and $\left\{W_{n}\right\}$ are sequences of finite-dimensional subspaces of $E$ and $W$, respectively, $\bigcup_{n=1}^{\infty} E_{n}$ is dense in $E$, and for each $n, Q_{n}$ is a projection of $W$ onto $W_{n}$. If $D \subseteq E$, then a mapping $h: D \rightarrow E$ will be called approximation-proper with respect to $\Gamma$ provided that

(i) $h^{-1}(0)$ is locally compact; and

(ii) for each $n,\left.h_{n} \equiv Q_{n} h\right|_{D \cap E_{n}}$ is continuous, and whenever $\left\{n_{k}\right\}$ is an increasing sequence of integers with $\left\{z_{n_{k}}\right\} \subseteq D$ bounded, $z_{n_{k}} \in D \cap E_{n_{k}}$ for each $k$, and $\left\{h_{n_{k}}\left(z_{n_{k}}\right)\right\} \rightarrow 0$, then $\left\{z_{n_{k}}\right\}$ has a subsequence which converges to $z \in D$ and $h(z)=0$.

REMARK 3.1. The above definition is a very slight variant of the customary definition of approximation-properness, abbreviated as A-properness (see Petryshyn [19]); in [19] condition (i) is not prescribed, condition (ii) holds where $h$ is replaced by $h-y$ for each $y \in Y$, and the approximation scheme is somewhat different. When $E=W, E_{n}=W_{n}$ for each $n$, and $\left\{Q_{n}(w)\right\} \rightarrow w$ for each $w \in W$, then $h \equiv I-C: D \rightarrow W$ is A-proper with respect to $\Gamma$ if $C$ is compact and $D$ is closed. 
Thus if $W$ is a Banach space with a Schauder basis, compact perturbations of the identity, defined on closed sets, are A-proper [19]. However, A-proper mappings form a much wider class than this particular subclass (see [19] and also our present $\S 5)$.

In the case when $\Gamma$ has the property that $\operatorname{dim} E_{n}=\operatorname{dim} W_{n}$ for each $n, 0 \subseteq E$ is bounded, and $h: \bar{O} \rightarrow W$ is A-proper with respect to $\Gamma$ with $0 \notin h(\partial \mathcal{O})$, there is defined by Browder and Petryshyn [8] the notion of topological degree of $h$ over $O$ at 0 . The definition is as follows. Letting $Z$ denote the integers, and $Z^{+}=Z \cup\{ \pm \infty\}, \operatorname{deg}(h, 0,0)$ is a subset of $Z^{+}$defined as follows: If $k \in Z$, then $k \in \operatorname{deg}(h, 0,0)$ provided that $\operatorname{deg}\left(h_{n}, E_{n} \cap 0,0\right)=k$ for infinitely many $n$; $+\infty(-\infty) \in \operatorname{deg}(h, 0,0)$ provided that there is an increasing sequence of integers $\left\langle n_{k}\right\rangle$ such that $\left\{\operatorname{deg}\left(h_{n_{k}}, E_{n_{k}} \cap 0,0\right)\right\}$ is unbounded above (below). In the above, each $E_{n}$ and $W_{n}$ is given an orientation, and then $\operatorname{deg}\left(h_{n}, E_{n} \cap 0,0\right)$ is the Brouwer degree with respect to this orientation provided that $0 \notin h_{n}\left(\partial\left(O \cap E_{n}\right)\right)$. When $O \subseteq E$ is unbounded but $h^{-1}(0)$ is bounded, one lets $\operatorname{deg}(h, O, 0)=\operatorname{deg}(h, \mathcal{U}, 0)$, where $\mathcal{U}$ is any bounded neighborhood of $h^{-1}(0)$ in 0 .

Now let $X$ and $Y$ be Banach spaces with $\left\{\left\langle X_{n}\right\rangle,\left\langle Y_{n}\right\rangle,\left\langle Q_{n}\right\rangle\right\}$ an approximation scheme for maps from $X$ to $Y$ such that $\operatorname{dim} X_{n}=\operatorname{dim} Y_{n}$ for each $n$. Let $D \subseteq \mathbf{R}^{m} \times$ $X$ and $f: D \rightarrow X$ be A-proper with respect to the scheme $\left\langle\left\{\mathbf{R}^{m} \times X_{n}\right\},\left\{Y_{n}\right\},\left\{Q_{n}\right\}\right\rangle$. Then it is clear that if $g: D \rightarrow \mathbf{R}^{m}$ is continuous and bounded, the mapping $(g \times f) \circ \Delta: D \rightarrow \mathbf{R}^{m} \times Y$ is A-proper with respect to the scheme $\left\langle\left\{\mathbf{R}^{m} \times X_{n}\right\},\left\{\mathbf{R}^{m} \times\right.\right.$ $\left.\left.Y_{n}\right\},\left\{\hat{Q}_{n}\right\}\right)$, where $\hat{Q}_{n}(\lambda, y)=\left(\lambda, Q_{n}(y)\right)$ for $\lambda \in \mathbf{R}^{m}, y \in Y$ and $n \in N$. (Recall that $\Delta$ is the diagonal map.)

When $O \subseteq \mathbf{R}^{m} \times X$ is open and $f: \bar{O} \rightarrow Y$ is A-proper with respect to $\left\langle\left\{\mathbf{R}^{m} \times\right.\right.$ $\left.\left.X_{n}\right\},\left\{Y_{n}\right\},\left\{Q_{n}\right\}\right\rangle$, a continuous mapping $g: \bar{\emptyset} \rightarrow \mathbf{R}^{m}$ is called a complement for $f$ on $\bar{O}$ provided that $\operatorname{deg}((g \times f) \circ \Delta, O, 0) \neq\{0\}$, where here we use the A-proper degree defined above.

Theorems 1.1 and 1.2 are valid with the definition of complement introduced above. The proofs of these theorems proceed in exactly the same way for both definitions (see $\S 4$ ). Moreover, the framework of this present section allows broader applications (see §5).

We will need the following variant of Remark 2.1.

Proposition 3.1. Let $X$ and $Y$ be Banach spaces with $\left\{\left\langle X_{n}\right\rangle,\left\langle Y_{n}\right\rangle,\left\langle Q_{n}\right\rangle\right\}$ an approximation scheme for mappings from $X$ to $Y$ such that $\operatorname{dim} X_{n}=\operatorname{dim} Y_{n}$ for each $n$. Suppose $O \subseteq \mathbf{R}^{m} \times X$ is open and $f: \bar{O} \rightarrow Y$ is A-proper with respect to $\left\langle\left\{\mathbf{R}^{m} \times X_{n}\right\},\left\{Y_{n}\right\},\left\{Q_{n}\right\}\right\rangle$. Let $\left(\lambda_{0}, x_{0}\right) \in \mathbf{R}^{m} \times X$, with $0_{\lambda_{0}}=\left\{x \in X \mid\left(\lambda_{0}, x\right) \in O\right\}$ and $f_{\lambda_{0}}=\left.f\right|_{\left\{\lambda_{0}\right\} \times O_{\lambda_{0}}}$. If $\operatorname{deg}\left(f_{\lambda_{0}}, O_{\lambda_{0}}, 0\right) \neq\{0\}$, then $f$ is complemented on $O$ by $g: \bar{O} \rightarrow \mathbf{R}^{m}$ defined by $g(\lambda, x)=\lambda-\lambda_{0}$.

PROOF. First of all observe that $f_{\lambda_{0}}: O_{\lambda_{0}} \subseteq X \rightarrow Y$ is A-proper with respect to $\left\langle\left\{X_{n}\right\},\left\{Y_{n}\right\},\left\{Q_{n}\right\}\right\rangle$. Since $\operatorname{deg}\left(f_{\lambda_{0}}, O_{\lambda_{0}}, 0\right) \neq\{0\}$, we may select an increasing sequence of integers, $\left\langle n_{k}\right\rangle$, such that $\operatorname{deg}\left(f_{\lambda_{0}}^{n_{k}}, O_{\lambda_{0}}^{n_{k}}, 0\right) \neq 0$, for each $k$, where $O_{\lambda_{0}}^{n_{k}}=$ $O_{\lambda_{0}} \cap X_{n_{k}}$ and $f_{\lambda_{0}}^{n_{k}}$ is the restriction of $Q_{n_{k}} f_{\lambda_{0}}$ to $O_{\lambda_{0}}^{n_{k}}$ for each $k$. Let $g_{n_{k}}$ be the restriction of $g$ to $O \cap X_{n_{k}}$.

It follows from Corollary 2.2 that $g_{n_{k}}$ is a complement for $f_{n_{k}}$ on $O_{n_{k}}$, where $O_{n_{k}}=\mathcal{O} \cap\left(\mathbf{R}^{m} \times X_{n_{k}}\right)$ and $f_{n_{k}}$ and $g_{n_{k}}$ are the restrictions of $Q_{n_{k} f}$ and $Q_{n_{k} g}$, 
respectively, to $O_{n_{k}}$. Thus

$$
\operatorname{deg}\left(((g \times f) \circ \Delta)_{n_{k}}, O_{n_{k}}, 0\right) \neq 0 \text { for each } k .
$$

From the definition of the A-proper degree it follows that

$$
\operatorname{deg}((g \circ f) \circ \Delta, 0,0) \neq\{0\} .
$$

REMARK 3.2. In the definition of A-properness one does not need the assumption that $h^{-1}(0)$ is locally compact in order for the degree to be well defined. Moreover when the restriction of $h$ to each closed bounded set is proper, as follows from condition (ii) in Definition 3.1 if $h$ is continuous and $\Gamma$ satisfies further conditions (see [19]), then $h^{-1}(0)$ is locally compact. The class of maps described in Definition 3.1 is what is needed for our present purposes. To prove a particular mapping is A-proper with respect to a scheme $\Gamma$ requires information about $f$ which is independent of $\Gamma$ together with further assumptions on $\Gamma$.

4. We will now prove Theorems 1.1 and 1.2. First we introduce some notation. For any pair of topological spaces $(V, W)$ with $W \subset V$ and positive integer $m$, $H^{m}(V, W)\left(H_{m}(V, W)\right)$ will denote the $m$ th singular cohomology (homology) groups with integral coefficients. When, in addition, $V$ and $W$ are normal, $\breve{H}^{m}(V, W)$ will denote the $m$ th Cech cohomology group. Finally, when $V$ and $W$ are locally compact, $\check{H}_{c}^{m}(V, W)$ will denote the $m$ th Cech cohomology group with compact supports.

We will use various properties of fundamental class, the Kronecker product and the cup product; these may be found in Dold [9].

Since our primary goal is to obtain information on the dimension of the set of zeros of a nonlinear operator, let us record explicitly the basic connection between cohomology and dimension through which our results will be obtained. A proof of the following proposition may be found in [10].

Proposition 4.1. Let $A$ be a normal topological space:

(i) If $\operatorname{dim}(A)<m$, then for each closed subspace $C$ of $A, \check{H}^{m}(A, C)=0$.

(ii) If $A$ is locally compact and $\operatorname{dim}(A)<m$, then $\breve{H}_{c}^{m}(\mathcal{U})=0$ for every open subset $\mathcal{U}$ of $A$.

We wish to prove Theorems 1.1 and 1.2 as stated in the introduction and their counterparts for approximation-proper mappings. For brevity, we do so simultaneously. Hence, when we write that $f: 0 \subseteq \mathbf{R}^{m} \times X \rightarrow Y$ is complemented by $g: \bar{O} \rightarrow \mathbf{R}^{m}$ we mean either that $X=Y$ and $f$ is a compact perturbation of the projection onto $X$ or that there is a suitable projection scheme with respect to which $f$ is approximation-proper.

Proposition 4.2. Let $X$ and $Y$ be Banach spaces, $O \subseteq \mathbf{R}^{m} \times X$ be open and bounded, and $f: \bar{O} \rightarrow Y$ be complemented by $g: \bar{O} \rightarrow \mathbf{R}^{m}$. Then the homomorphism induced in Cech cohomology by $g:\left(f^{-1}(0), f^{-1}(0) \cap \partial 0\right) \rightarrow\left(\mathbf{R}^{m}, \mathbf{R}^{m}-0\right)$ is nontrivial.

Proof. We first of all consider the case when $X=Y=\mathbf{R}^{n}$. Then $\mathbf{R}^{m} \times X=$ $\mathbf{R}^{n+m}$. Let $S=\{x \in \bar{O} \mid f(x)=0\}$ and $\dot{S}=S \cap \partial 0$. Let $V \subseteq \bar{O}$ be an open neighborhood (relative to $\bar{O}$ ) of $S$, and let $W \subset V$ be any neighborhood of $\dot{S}$ such 
that $g^{-1}(0) \cap W=\varnothing$. We will first show that the homomorphism induced in the singular cohomology by $g:(V, W) \rightarrow\left(\mathbf{R}^{m}, \mathbf{R}^{m}-0\right)$ is nontrivial.

We denote by $e_{k}$ a generator of $H^{k}\left(\mathbf{R}^{k}, \mathbf{R}^{k}-0\right)$.

Note that if we let $Z=V-S$, then $f:(V, Z) \rightarrow\left(\mathbf{R}^{n}, \mathbf{R}^{n}-0\right)$ induces

$$
f^{*}: H^{n}\left(\mathbf{R}^{n}, \mathbf{R}^{n}-0\right) \rightarrow H^{n}(V, Z),
$$

so it will suffice to show that the cup product $f^{*}\left(e_{n}\right) \cup g^{*}\left(e_{m}\right)$ is nontrivial in $H^{n+m}(V, W \cup Z)$.

Observe that

$$
\eta \equiv g^{*}\left(e_{n}\right) \cup f^{*}\left(e_{m}\right)=\Delta^{*}\left(g^{*}\left(e_{n}\right) \times f^{*}\left(e_{m}\right)\right)=[(g \times f) \circ \Delta]^{*}\left(e_{n+m}\right),
$$

where $\Delta:(V, W \cup Z) \rightarrow(V \times V,(W \times V) \cup(V \times Z))$ is the diagonal (see Dold [7, Chapter 7, §8]).

Let $K=V-(W \cup Z)$ and $V^{\prime}=V \cap 0$. Then $K$ is compact, $V^{\prime}$ is an open subset of $\mathbf{R}^{n+m}$, and $K \subseteq S-\dot{S} \subset V^{\prime}$. Hence, we have the inclusion of pairs $i:\left(V^{\prime}, V^{\prime}-K\right) \rightarrow(V, W \cup Z)$. If we let

$$
G=(g \times f) \circ \Delta:\left(V^{\prime}, V^{\prime}-K\right) \rightarrow\left(\mathbf{R}^{n+m}, \mathbf{R}^{n+m}-0\right),
$$

from (4.1) we see that $i^{*}(\eta)=G^{*}\left(e_{n+m}\right)$.

On the other hand, since $G^{-1}(0) \subset K$, if $O_{K} \in H_{n+m}\left(V^{\prime}, V^{\prime}-K\right)$ is the fundamental class around $K$ and $O_{n+m}$ denotes the fundamental class around $\{0\}$ in $\mathbf{R}^{n+m}$, then

$$
G_{*}\left(O_{K}\right)=\operatorname{deg}\left(G, V^{\prime}, 0\right) \cdot O_{n+m} \text { in } H_{n+m}\left(\mathbf{R}^{n+m}, \mathbf{R}^{n+m}-0\right)
$$

(see Proposition 5.5 of $[9]$ ).

By taking the Kronecker product of $i^{*}(\eta)$ with $O_{K}$ we get

$$
\left\langle i^{*}(\eta), O_{K}\right\rangle=\left\langle G^{*}\left(e_{n+m}\right), O_{K}\right\rangle=\left\langle e_{n+m}, G_{*}\left(O_{K}\right)\right\rangle=\operatorname{deg}\left(G, V^{\prime}, 0\right),
$$

since $\left\langle e_{n+m}, O_{n+m}\right\rangle=1$. Thus $\eta \neq 0$.

Now suppose $\bar{O}$ is an E.N.R. It is well known that the Cech cohomology of the compact pair $(S, \dot{S})$ can be evaluated as $\underset{\longrightarrow}{\longrightarrow} H^{*}(V, W)$, where $(V, W)$ ranges over all neighborhood pairs of $(S, \dot{S})$ (see [9]). However the family of neighborhood pairs $(V, W)$ with $g^{-1}(0) \cap W=\varnothing$ is cofinal, and since

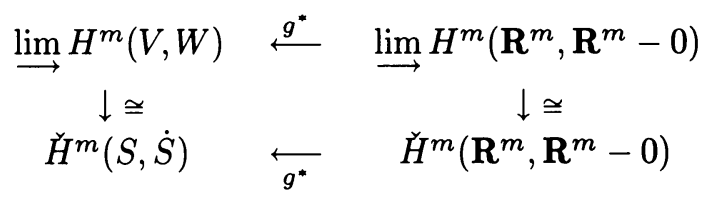

is commutative, it follows that $g^{*}\left(e_{m}\right) \in \check{H}^{m}(S, \dot{S})$ is nonzero. If $\mathcal{O}$ is any open and bounded subset of $\mathbf{R}^{m} \times \mathbf{R}^{n}$, the assertion follows from the above by considering $\bar{O}$ as an intersection of a nested sequence of E.N.R.'s, and then invoking the continuity of Cech cohomology.

We now consider the infinite-dimensional case. As before, let $(V, W)$ be any neighborhood pair of $(S, \dot{S})$ such that $g^{-1}(0) \cap W=\varnothing$.

First consider the case when $X=Y$ and $f(\lambda, x)=x-C(\lambda, x)$ for $(\lambda, x) \in \overline{0}$, where $C(\overline{0})$ has compact closure. 
We claim that we can find a finite-dimensional subspace $H$ of $X$ and a continuous mapping $h: \bar{O} \cap\left(\mathbf{R}^{m} \times H\right) \rightarrow H$ such that $h^{-1}(0) \cap \bar{O}_{H} \subseteq V, h^{-1}(0) \cap \partial O_{H} \subseteq W$ and $\left.g\right|_{\bar{O}_{H}}$ complements $h$ if $O_{H}=O \cap\left(\mathbf{R}^{m} \times H\right)$. Assume, for the moment, that this claim is justified.

Letting $S_{H}=h^{-1}(0) \cap \bar{\mho}_{H}$ and $\dot{S}_{H}=S_{H} \cap \partial O_{H}$, we see from the finitedimensional case just proven that $g:\left(S_{H}, \dot{S}_{H}\right) \rightarrow\left(\mathbf{R}^{m}, \mathbf{R}^{m}-0\right)$ induces a nontrivial homomorphism in cohomology. Moreover, the following diagram is commutative:

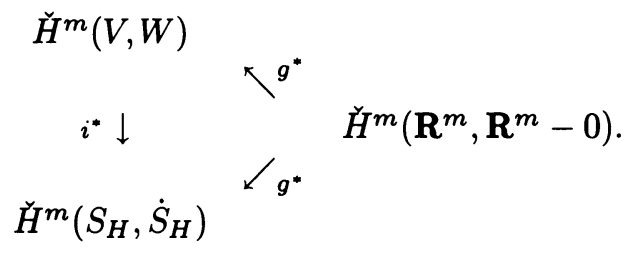

Consequently, $g:(V, W) \rightarrow\left(\mathbf{R}^{m}, \mathbf{R}^{m}-0\right)$ induces a nontrivial element in cohomology. But, by tautness (see [22, §6, Chapter 6]), $\check{H}^{m}(S, \dot{S})=\lim _{\longrightarrow} \check{H}^{m}(V, W)$, where $(V, W)$ ranges over neighborhood pairs of $(S, \dot{S})$. Since those pairs with the additional property that $g^{-1}(0) \cap W=\varnothing$ are cofinal, it follows that $g:(S, \dot{S}) \rightarrow$ $\left(\mathbf{R}^{m}, \mathbf{R}^{m}-0\right)$ induces a nontrivial homomorphism in cohomology.

It remains to verify the approximation claim. Since $O$ is bounded and $C$ is compact, there is an $\varepsilon>0$ such that $\|(g(\lambda, x), f(\lambda, x))\| \geq \varepsilon$ if $(\lambda, x) \in \partial O$, and $\|f(\lambda, x)\| \geq \varepsilon$ if $x \in \bar{O}-V$ or $x \in \partial \mathcal{O}-W$. On the other hand, by the basic approximation property for compact mappings we may find a finite-dimensional space, $H$, and $\psi: \bar{O} \rightarrow H$ such that $\|\psi(\lambda, x)-C(\lambda, x)\|<\varepsilon$ for all $(\lambda, x) \in \overline{0}$. From the definition of the Leray-Schauder degree it follows that if $h(\lambda, x)=x-\psi(\lambda, x)$ for $(\lambda, x) \in \bar{O}_{H}$, then $h$ is complemented by $\left.g\right|_{\bar{O}_{H}}$. It is also clear, by our choice of $\varepsilon$, that $h^{-1}(0) \subseteq V$ and $h^{-1}(0) \cap \partial O_{H} \subseteq W$.

Now consider the situation described in the previous section. Let $X$ and $Y$ be Banach spaces with $\Gamma=\left\langle\left\{\mathbf{R}^{m} \times X_{n}\right\},\left\{Y_{n}\right\},\left\{Q_{n}\right\}\right\rangle$ a projection scheme for mappings from $\mathbf{R}^{m} \times X$ to $Y$ with respect to which $f$ is A-proper and complemented by $g$.

By an argument exactly the same as in the first case, to conclude the proof in this case it will suffice to find some $k_{0} \in \mathbf{N}$ such that, if $f_{k}=\left.Q_{k} f\right|_{0 \cap\left(\mathbf{R}^{m} \times X_{k}\right)}$, then $f_{k_{0}}^{-1}(0) \subseteq V, f_{k_{0}}^{-1}(0) \cap \partial O \subseteq W$, and $\operatorname{deg}\left(\left(f_{k_{0}}, g\right), O \cap\left(\mathbf{R}^{m} \times X_{k_{0}}\right), 0\right) \neq 0$.

Using the definition of the A-proper degree, we may, by taking subsequences if necessary, assume that $\operatorname{deg}\left(\left(f_{k}, g\right), O \cap\left(\mathbf{R}^{m} \times X_{k}\right), 0\right) \neq 0$ for all $k$.

Let $S_{k}=f_{k}^{-1}(0)$. We claim we can choose $k^{1}$ such that $S_{k} \subseteq V$ if $k \geq k^{1}$. Indeed, if this is not the case, then we can choose an increasing sequence of integers, $\left\{n_{k}\right\}$, and $x_{n_{k}} \in S_{n_{k}}-V$ for each $k$. From the definition of A-properness it follows that there is a subsequence of $\left\{x_{n_{k}}\right\}$ which converges to $x \in \bar{O}$ and $f(x)=0$. But $x \notin S$ since $V$ is a neighborhood of $S$. This contradiction shows that a $k^{1}$ with the above property may be chosen. The same argument allows us to choose $k_{0} \geq k^{1}$ with

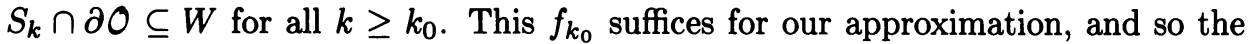
second case is proven.

Given $\xi \in \breve{H}_{c}^{m}(A)$ and $D \subseteq A$ with $D$ closed, by $\left.\xi\right|_{D}$ we denote $i^{*}(\xi)$, where $i: D \rightarrow A$ is the inclusion. We now wish to prove that if $\xi \in \breve{H}_{c}^{m}(A), \xi \neq 0$, then $\xi$ is supported by a particular minimal subset of $A$ (see also [4, Chapter 2, Corollary 10.6]). 
Proposition 4.3. Let $A$ be a locally compact topological space. Suppose $\xi \in$ $\check{H}_{c}^{m}(A)$ is nontrivial. Then there exists a closed, connected subset $C$ of $A$ which has dimension at each point at least $m$, is such that $\left.\xi\right|_{C} \neq 0$, and whenever $C^{\prime}$ is a proper and closed subset of $C,\left.\xi\right|_{C^{\prime}}=0$.

ProOF. Let $A^{+}$denote the one-point compactification of $A$ obtained by adjoining $\infty$. There is a natural isomorphism $\eta$ : $\check{H}_{c}^{m}(A) \rightarrow \check{H}^{m}\left(A^{+},\{\infty\}\right)$. Since $\eta(\xi)$ is nontrivial, by Proposition 2.2 of [16] we may choose a connected subset $C^{\prime}$ of $A^{+}$such that $\left.\eta(\xi)\right|_{C^{\prime}}$ is nontrivial in $\check{H}^{m}\left(C^{\prime},\{\infty\}\right)$ and such that whenever $C^{\prime \prime}$ is a proper closed subset of $C^{\prime}$, then $\left.\eta(\xi)\right|_{C^{\prime \prime}}=0$.

If we let $C=C^{\prime}-\{\infty\}$, then again using Proposition 2.2 of $[\mathbf{1 6}], C$ is connected and from the naturality of $\eta,\left.\xi\right|_{C} \neq 0$. Moreover, $C$ is minimal because $C^{\prime}$ is.

To prove the dimension conclusion, we let $p \in C$ and let $W$ be any open neighborhood of $p$ in $C$.

Consider the exact sequence $\breve{H}_{c}^{m}(W) \rightarrow \check{H}_{c}^{m}(C) \stackrel{i^{*}}{\rightarrow} \check{H}_{c}^{m}(C-W)$. From the minimality of $C$ it follows that $i^{*}\left(\left.\xi\right|_{C}\right)=0$, and from the exactness of the sequence it follows that $\left.\xi\right|_{C}$ must be the image of some nontrivial element of $\breve{H}_{c}^{m}(W)$. From Proposition 4.1(ii) we conclude that $\operatorname{dim}(W) \geq m$.

ProOF OF THEOREM 1.1. Recall that $\check{H}_{c}^{m}(S, \dot{S})=\underline{\lim } \breve{H}^{m}(S, T)$, where $T$ ranges over all cobounded neighborhoods of $\dot{S}$ (cf. [22]). Let $T_{0}=S-g^{-1}(0)$, and let $j_{T_{0}}: \breve{H}^{m}\left(S, T_{0}\right) \rightarrow \breve{H}_{c}^{m}(S, \dot{S})$ be the natural map into the direct limit. We wish to show that $j_{T_{0}}\left(g^{*}\left(e_{m}\right)\right) \neq 0$, and to do so it will suffice to verify that for each cobounded neighborhood $T$ of $\dot{S}$ with $T \subset T_{0}$ we have that $\left.g^{*}\left(e_{m}\right)\right|_{(S, T)}=$ $\left(\left.g\right|_{(S, T)}\right)^{*}\left(e_{m}\right)$ is nontrivial.

Let $T$ be such a neighborhood of $\dot{S}$. We may choose an open ball $B$ in $X$ containing $S-T$. Let $\mathcal{U}=O \cap B$. By the excision property for topological degree, $\operatorname{deg}(F, O, 0)=\operatorname{deg}(F, \mathcal{U}, 0)$. Hence, by Proposition 4.2, we conclude that $g:(S \cap \bar{U}, S \cap \partial \mathcal{U}) \rightarrow\left(\mathbf{R}^{m}, \mathbf{R}^{m}-0\right)$ induces a nontrivial map in cohomology. But $(S \cap \overline{\mathcal{U}}, S \cap \partial \mathcal{U}) \subseteq(S, T)$, so that the commutativity of

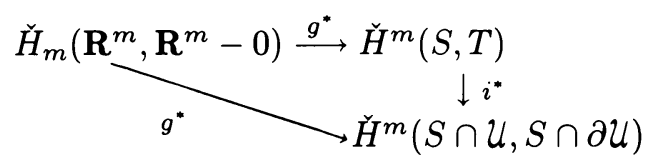

implies that $g:(S, T) \rightarrow\left(\mathbf{R}^{m}, \mathbf{R}^{m}-0\right)$ induces a nontrivial homomorphism in cohomology. Let $\xi$ denote $j_{T_{0}}\left(g^{*}\left(e_{m}\right)\right)$; then $\xi \neq 0$ in $H_{c}^{m}(S, \dot{S})$.

The isomorphism $\gamma: \breve{H}_{c}^{m}(S, \dot{S}) \rightarrow \check{H}_{c}^{m}(S-\dot{S})$ yields a nontrivial element $\gamma(\xi)$ in $\breve{H}_{c}^{m}(S-\dot{S})$. Hence, by Proposition 4.3 there is a connected subset $C_{0}$ of $S-\dot{S}$, whose dimension at each point is at least $m$ and such that $\left.\gamma(\xi)\right|_{C_{0}}$ is nontrivial. Let $C$ be the closure of $C_{0}$ in $S$.

Clearly $C$ is a connected subset of $S$ such that at each point $p \in C-\dot{S}$ it has dimension at least $m$. It remains to prove the global properties of $C$. 
Consider the following commutative diagram, where $A$ is any cobounded subset of $C$, containing $\dot{C}$, with $A \cap g^{-1}(0)=\varnothing$ :

$$
\begin{array}{rlccccc}
H^{m}\left(\mathbf{R}^{m}, \mathbf{R}^{m}-0\right) \stackrel{g^{*}}{\rightarrow} & H^{m}\left(S, T_{0}\right) & \stackrel{j_{T_{\rho}}}{\rightarrow} & \check{H}_{c}^{m}(S, \dot{S}) & \stackrel{\gamma}{\rightarrow} & \check{H}_{c}^{m}(S-\dot{S}) \\
g^{*} \searrow & \begin{array}{c}
\downarrow i^{*} \\
\end{array} & & & \downarrow \check{H}^{*} & & \\
& \check{H}^{m}(C, A) & \overrightarrow{j_{A}} & \check{H}_{c}^{m}(C, \dot{C}) & \underset{\gamma}{\rightarrow} & \check{H}_{c}^{m}\left(C_{0}\right)
\end{array}
$$

From inspection of this diagram and the construction of $C$ it follows that $g:(C, A) \rightarrow$ $\left(\mathbf{R}^{m}, \mathbf{R}^{m}-0\right)$ induces a nontrivial homomorphism in cohomology.

In particular, $C \cap g^{-1}(0) \neq \varnothing$, since otherwise $g(C) \subset \mathbf{R}^{m}-0$, in which case $g:(C, A) \rightarrow\left(\mathbf{R}^{m}, \mathbf{R}^{m}-0\right)$ induces the trivial homomorphism for any $A$ as above.

If $C$ is unbounded, the proof is complete. So assume that $C$ is bounded. In this case $C$ is compact and $\dot{C} \equiv C \cap \partial O$ is a cobounded subset of $C$, and so from the above $g^{*}: \breve{H}^{m}\left(\mathbf{R}^{m}, \mathbf{R}^{m}-0\right) \rightarrow \breve{H}^{m}(C, \dot{C})$ is nontrivial.

First consider the case when $m=1$. We claim $g: C \cap \partial \mathcal{U} \rightarrow \mathbf{R}-0$ is essential; i.e., it assumes both positive and negative values. Indeed, if this were not so then $g:(C, \dot{C}) \rightarrow(\mathbf{R}, \mathbf{R}-0)$ could be deformed, as a map of pairs, via a linear homotopy to a constant map in contradiction to the nontriviality of $g^{*}\left(e_{1}\right)$.

Finally, consider $m>1$. We have the following commutative diagram, with the bottom row being exact:

$$
\begin{aligned}
& \rightarrow \check{H}^{m-1}(\dot{C}) & \rightarrow \check{H}^{m}(C, \dot{C}) \\
& \uparrow_{g^{*}} & \\
0 & \rightarrow \check{H}^{m-1}\left(\mathbf{R}^{m}-0\right) & \rightarrow \check{H}^{m}\left(\mathbf{R}^{m}, \mathbf{R}^{m}-0\right) \rightarrow 0
\end{aligned}
$$

Consequently, $\breve{H}^{m-1}(\dot{C})$ is nontrivial, and so $\dot{C}$ has dimension at least $(m-1)$, and $g: \dot{C} \rightarrow \mathbf{R}^{m}-0$ is essential.

PROOF OF THEOREM 1.2. Since $\left.g\right|_{S}$ is proper, and $g:(S, \dot{S}) \rightarrow\left(\mathbf{R}^{m}, \mathbf{R}^{m}-0\right)$, it induces

$$
g^{*}: \check{H}^{m}\left(\mathbf{R}^{m}, \mathbf{R}^{m}-0\right) \cong \check{H}_{c}^{m}\left(\mathbf{R}^{m}, \mathbf{R}^{m}-0\right) \rightarrow \check{H}_{c}^{m}(S, \dot{S})
$$

From the argument at the beginning of the proof of Theorem 1.1, and since we now have assumed $\left.g\right|_{S}$ is proper, it follows that the element $\xi$ defined in that proof coincides with $g^{*}\left(e_{m}\right) \in \check{H}_{c}^{m}(S, \dot{S})$.

Once more invoking the cohomological characterization of dimension and using the assumption that the covering dimension of $\dot{S}$ is less than $m-1$, we conclude that $\check{H}_{c}^{m-1}(\dot{S})=0$. Thus, from the exactness of the sequence of the pair $(S, \dot{S})$, it follows that $\breve{H}_{c}^{m}(S, \dot{S}) \rightarrow \breve{H}_{c}^{m}(S)$ is injective.

Consider the following commutative diagram:

$$
\begin{array}{ccc}
\check{H}_{c}^{m}(S, \dot{S}) & \stackrel{\text { injective }}{\longrightarrow} & \check{H}_{c}^{m}(S) \\
g^{*} \uparrow & & g^{*} \uparrow \\
\check{H}^{m}\left(\mathbf{R}^{m}, \mathbf{R}^{m}-0\right) & \longrightarrow & \check{H}_{c}^{m}\left(\mathbf{R}^{m}\right)
\end{array}
$$

If $e \in \check{H}_{c}^{m}\left(\mathbf{R}^{m}\right)$ is the image of $e_{m}$ under the lower map, then $\xi^{\prime}=g^{*}(e) \in \check{H}_{c}^{m}(S)$ is nontrivial. Using Proposition 4.2 we may choose a connected subset $C$ of $S$ whose dimension at each point is at least $m$ and $\left.\xi^{\prime}\right|_{C}$ is nontrivial; $\left.\xi^{\prime}\right|_{C}$ is the image of $e_{m}$ under the homomorphism induced by $g: C \rightarrow \mathbf{R}^{m}$. Consequently, when considered among proper mappings, $g$ is essential. In particular, $g(C)=\mathbf{R}^{m}$. 
5. Here we wish to indicate the applicability of our previous results to some existence problems for partial differential equations. Two examples are considered. The first is an eigenvalue problem for a partial differential equation in generalized divergence form whose higher-order part satisfies the Leray-Lions conditions (see [14]). The second is an existence problem for a partial differential equation which is in the form of a nonlinear perturbation of a linear elliptic differential operator which generates, when acting between prescribed Sobolev spaces, a Fredholm operator of positive index. Let us first establish our notation.

Let $\Omega \subseteq \mathbf{R}^{n}$ be a bounded domain. For an $n$-tuple of nonnegative integers $\alpha=\left(\alpha_{1}, \ldots, \alpha_{n}\right)$, we let $D^{\alpha}=\prod_{j=1}^{n}\left(\partial / \partial x_{j}\right)^{\alpha_{j}}$, with the order of $D^{\alpha}$ being written as $|\alpha|=\sum_{j=1}^{n} \alpha_{j}$. When $k$ is a positive integer, and $1 \leq p<\infty$, the Sobolev space $W^{k, p}(\Omega)$ consists of those functions in $L^{p}(\Omega)$ whose distributional derivatives up to order $k$ lie in $L^{p}(\Omega)$. Then, if $1<p<\infty, W^{k, p}(\Omega)$, with norm $\|u\|^{p}=$ $\sum_{|\alpha| \leq k}\left\|D^{\alpha}(u)\right\|_{L^{p}}^{p}$, is a separable reflexive Banach space. We assume throughout that $\Omega$ is such that the Rellich compactness theorem and the Sobolev embedding theorems hold. By $\stackrel{\circ}{W}^{k, p}(\Omega)$ we will denote the closure in $W^{k, p}(\Omega)$ of the infinitely differentiable, compactly supported functions on $\Omega$.

Now, for $j$ a nonnegative integer, let $s_{j}$ be the number of $n$-tuples of nonnegative integers whose order does not exceed $j$. Then for $u \in W^{j, p}(\Omega)$ we define

$$
\xi_{j}(u): \Omega \rightarrow \mathbf{R}^{s_{j}}
$$

by $\left(\xi_{j}(u)(x)\right)_{\alpha}=D^{\alpha} u(x)$ for $\alpha=\left(\alpha_{1}, \ldots, \alpha_{n}\right),|\alpha| \leq j, x \in \Omega$.

The $2 k$ th order quasilinear eigenvalue problem, in generalized divergence form depending on a parameter $\lambda \in \mathbf{R}^{m}$, which we wish to consider is

$$
\begin{aligned}
& \sum_{|\alpha| \leq k}(-1)^{|\alpha|} D^{\alpha}\left(A_{\alpha}\left(x, \xi_{k}(u)(x)\right)\right) \\
& \quad=\sum_{|\beta| \leq k-1}(-1)^{|\beta|} D^{\beta}\left(B_{\beta}\left(\lambda, x, \xi_{k-1}(u)(x)\right)\right), \quad x \in \Omega,
\end{aligned}
$$

and we specify the boundary conditions by insisting that $u \in V$, where $V$ is a prescribed subspace of $W^{k, p}(\Omega)$ which contains $\stackrel{\circ}{W}^{k, p}(\Omega)$.

We shall look for a variational solution of (5.1). That is, we seek $u \in V$ such that

$$
\begin{aligned}
& \sum_{|\alpha| \leq k} \int_{\Omega} A_{\alpha}\left(x, \xi_{k}(u)(x)\right) D^{\alpha}(\varphi) d x \\
& \quad=\sum_{|\beta| \leq k-1} B_{\beta}\left(\lambda, x, \xi_{k-1}(u)(x)\right) D^{\beta}(\varphi)(x) d x \quad \text { for all } \varphi \in V .
\end{aligned}
$$

We now need to make the analytical assumptions on the $A_{\alpha}$ 's and $B_{\beta}$ 's in order to formulate (5.2) in our abstract setting. Roughly speaking, if the $A_{\alpha}$ 's and $B_{\beta}$ 's have polynomial growth, then we can formulate the problem in a suitable $V \subseteq W^{k, p}$, $p$ being determined by the growth. The right-hand side of (5.2) will correspond to a completely continuous operator. If the left-hand side corresponds to a linear bijection, the problem can be formulated in the Leray-Schauder context. However, very general left-hand sides can be formulated within the A-proper context. To do 
so, we impose the following conditions, where $1<p<\infty$ :

$A_{\alpha}: \Omega \times \mathbf{R}^{s_{k}} \rightarrow \mathbf{R}, B_{\beta}: \mathbf{R}^{m} \times \Omega \times \mathbf{R}^{s_{k-1}} \rightarrow \mathbf{R}$, for $|\alpha| \leq k,|\beta| \leq k-1$, are such that (1) $A_{\alpha}(x, \xi)$ is measurable in $x$ for fixed $\xi \in \mathbf{R}^{s_{k}}$ and continuous in $\xi$ for fixed $x$ in $\Omega,(2) B_{\beta}$ is measurable in $x$ for fixed $\lambda \in \mathbf{R}^{m}, \xi \in \mathbf{R}^{s_{k-1}}$, and continuous in $\lambda$ and $\xi$ for fixed $x$ in $\Omega$.

(5.4) There exist functions $\varphi, \psi \in L^{\infty}(\Omega)$ such that $\left|A_{\alpha}(x, \xi)\right| \leq \varphi(x)+$ $\psi(x)|\xi|^{p-1}, x \in \Omega, \xi \in \mathbf{R}^{s_{k}},\left|B_{\beta}(\lambda, x, \xi)\right| \leq \varphi(x)+\psi(x)|\xi|^{p-1-\varepsilon}$ $x \in \Omega, \xi \in \mathbf{R}^{s_{k-1}}$, where $\varepsilon \geq 0$.

(5.5) If $\xi=(\eta, \nu)$ denotes the division of $\xi \in \mathbf{R}^{s_{k}}$ into $k$ th-order components, represented by $\nu$, and lower-order components, represented by $\eta$, and if $\nu^{\alpha}$ is the $\alpha$ th component of $\nu$ for $|\alpha|=k$, then

$$
\sum_{|\alpha|=k}\left[A_{\alpha}(x, \eta, \nu)-A_{\alpha}(x, \eta, \bar{\nu})\right]\left[\nu^{\alpha}-\bar{\nu}^{\alpha}\right]>0,
$$

whenever $(\eta, \nu),(\eta, \bar{\nu}) \in \mathbf{R}^{s_{k}}$ and $\nu \neq \bar{\nu}$.

(5.6) There exist constants $C_{0}>0$ and $C_{1}$ such that for all $x \in \Omega$, $\xi \in \mathbf{R}^{s_{k}}$,

$$
\sum_{|\alpha| \leq k} A_{\alpha}(x, \xi) \xi^{\alpha} \geq C_{0}|\xi|^{p}-C_{1}
$$

Now assumptions (5.3) and (5.4) guarantee that for each $|\alpha| \leq k,|\beta| \leq k-1$, and $u \in W^{k, p}(\Omega), A_{\alpha}\left(\cdot, \xi_{k}(u)\right)$ and $B_{\beta}\left(\cdot, \xi_{k-1}(u)\right)$ are in $L^{q}(\Omega), p^{-1}+q^{-1}=1$. Thus, letting $V^{*}$ denote the dual of $V$, and letting $($,$) denote the pairing between$ $V^{*}$ and $V$, we may define $A(u) \in V^{*}, B(\lambda, u) \in V^{*}$, for $u \in V, \lambda \in \mathbf{R}^{m}$, by

$$
\begin{aligned}
(A(u), \varphi) & =\sum_{|\alpha| \leq k} \int_{\Omega} A_{\alpha}\left(x, \xi_{k}(u)(x)\right) D^{\alpha} \varphi(x) d x \quad \text { for } \varphi \in V, \\
(B(\lambda, u), \varphi) & =\sum_{|\beta| \leq k-1} \int_{\Omega} B_{\beta}\left(\lambda, x, \xi_{k-1}(u)(x)\right) D^{\beta} \varphi(x) d x \quad \text { for } \varphi \in V .
\end{aligned}
$$

Then it is clear that equation (5.2) is equivalent to

$$
A(u)=B(\lambda, u), \quad u \in V, \lambda \in \mathbf{R}^{m} .
$$

From (5.3), (5.4), and standard properties of the Nemytsky operator it follows that $A: V \rightarrow V^{*}$ and $B: \mathbf{R}^{m} \times V \rightarrow V^{*}$ are continuous and map bounded sets into bounded sets. Moreover, by the Rellich compactness theorem, $B$ maps weakly convergent sequences into strongly convergent sequences.

The crucial consequence of the Leray-Lions assumptions (5.5) and (5.6), which we will need in order to show that $A-B$ is an A-proper mapping, are stated in the following proposition. For a detailed proof see the appendix of $\S 1$ of [6], and also [14]. 
Proposition 5.1. Assume that the $A_{\alpha}$ 's of equation (5.1) satisfy (5.3)-(5.6). Let $A: V \rightarrow V^{*}$ be defined by (5.7). Then, if $\left\{v_{j}\right\} \subseteq V$ converges weakly to $v$ in $V$ and $\lim _{j \rightarrow \infty}\left(A\left(v_{j}\right)-A(v), v_{j}-v\right)=0$, it follows that $\left\{v_{j}\right\}$ converges strongly to $v$.

The property enjoyed by the operator in the above proposition is called property (S) in Browder [7], where its relevance to the convergence of certain generalized Galerkin methods is made clear. The basic idea of the following result may be found in [7]. Since our definitions are slightly different, and the proof is so short, we include a proof here.

Proposition 5.2. Assume the $A_{\alpha}$ 's and $B_{\beta}$ 's of equation (5.1) satisfy (5.3)(5.6). Let $A$ and $B$ be as defined by (5.7) and (5.8). Choose $\left\{V_{j}\right\}$ to be any increasing sequence of finite-dimensional subspaces of $V$ whose union is dense, and for each $j$ let $P_{j}$ be a linear projection of $V$ onto $V_{j}$. Then $A-B: \mathbf{R}^{m} \times V \rightarrow V^{*}$ is $A$ proper with respect to the approximation scheme $\Gamma=\left\langle\left\{\mathbf{R}^{m} \times V_{j}\right\},\left\{P_{j}^{*}\left(V_{j}\right)\right\},\left\{P_{j}^{*}\right\}\right\rangle$.

Proof. Since $V$ is a closed subspace of $L^{p}(\Omega), 1<p<\infty$, such a sequence $\left\{V_{j}\right\}$ may be chosen.

Suppose $\left\{n_{k}\right\}$ is an increasing sequence of positive integers, with $\left\{v_{n_{k}}\right\}$ bounded, $v_{n_{k}} \in V_{n_{k}}$ for each $k,\left\{\lambda_{n_{k}}\right\} \subseteq \mathbf{R}^{m}$ is bounded, and $\left\{P_{n_{k}}^{*} A\left(v_{n_{k}}\right)-P_{n_{k}}^{*} B\left(\lambda_{n_{k}}, v_{n_{k}}\right)\right\}$ $\rightarrow 0$. Now $V$ is reflexive, and hence has weakly sequentially compact closed balls. Thus, without loss of generality, we may assume $\left\{v_{n_{k}}\right\}$ converges weakly to $v \in V$, and $\left\{\lambda_{n_{k}}\right\} \rightarrow \lambda \in \mathbf{R}^{m}$. By the Rellich compactness theorem

$$
\left\{\xi_{k-1}\left(v_{n_{k}}\right)\right\} \rightarrow \xi_{k-1}(v) .
$$

Consequently, $\left\{B\left(\lambda_{n_{k}}, v_{n_{k}}\right)\right\} \rightarrow B(\lambda, v)$. From our choice of $\left\{V_{n}\right\}$ we may select $w_{n_{k}} \in V_{n_{k}}$ for each $k$, and $\left\{w_{n_{k}}\right\} \rightarrow v$.

Now, for each $k$,

$$
\begin{aligned}
\left(A\left(v_{n_{k}}\right)\right. & \left.-A(v), v_{n_{k}}-v\right) \\
= & \left(A\left(v_{n_{k}}\right), v_{n_{k}}-w_{n_{k}}\right)+\left(A\left(v_{n_{k}}\right), w_{n_{k}}-v\right)-\left(A(v), v_{n_{k}}-v\right) \\
= & \left(P_{n_{k}}^{*} A\left(v_{n_{k}}\right)-P_{n_{k}}^{*} B\left(\lambda_{n_{k}}, v_{n_{k}}\right), v_{n_{k}}-w_{n_{k}}\right)+\left(B\left(\lambda_{n_{k}}, v_{n_{k}}\right), v_{n_{k}}-w_{n_{k}}\right) \\
& +\left(A\left(v_{n_{k}}\right), w_{n_{k}}-v\right)-\left(A(v), v_{n_{k}}-v\right) .
\end{aligned}
$$

Since $\left\{v_{n_{k}}\right\}$ and $\left\{w_{n_{k}}\right\}$ converge weakly (respectively, strongly) to $v$ and $\left\{A\left(v_{n_{k}}\right)\right\}$ is bounded, each of the four sequences defined above converges to 0 .

Hence, from Proposition 5.1, it follows that $\left\{v_{n_{k}}\right\} \rightarrow v$. Also, $A-B$ is continuous, and so $A(v)-B(\lambda, v)=\lim _{k \rightarrow \infty}\left[A\left(v_{n_{k}}\right)-B\left(\lambda_{n_{k}}, v_{n_{k}}\right)\right]$.

We claim $A(v)-B(\lambda, v)=0$. Indeed, let $u \in V$ and choose $\left\{u_{n_{k}}\right\} \rightarrow u$ with $u_{n_{k}} \in V_{n_{k}}$ for each $k$. Then

$$
\begin{aligned}
0 & =\lim \left(P_{n_{k}}^{*} A\left(v_{n_{k}}\right)-P_{n_{k}}^{*} B\left(\lambda_{n_{k}}, v_{n_{k}}\right), u_{n_{k}}\right) \\
& =\lim \left(A\left(v_{n_{k}}\right)-B\left(\lambda_{n_{k}}, v_{n_{k}}\right), u_{n_{k}}\right)=(A(v)-B(\lambda, v), u) .
\end{aligned}
$$

Thus, $A(v)-B(\lambda, v)=0$.

Finally, from the reflexivity of $V$ and Proposition 5.1 it follows that the restriction of $A-B$ to any closed, bounded, subset of $\mathbf{R}^{m} \times V$ is proper. Thus $(A-B)^{-1}(0)$ is locally compact. 
We need one final assumption

(5.10) Either (5.4) holds with $\varepsilon>0$ or

$$
\sum_{|\beta| \leq k-1} B_{\beta}(\lambda, x, \xi) \xi^{\beta} \leq 0 \quad \text { for } \xi \in \mathbf{R}^{s_{k-1}}, \lambda \in \mathbf{R}^{m}, x \in \Omega .
$$

THEOREM 5.1. Suppose the $A_{\alpha}$ 's and $B_{\beta}$ 's of equation (5.1) satisfy assumptions (5.3)-(5.6) and (5.10). Then there exists a connected subset $C$ in $\mathbf{R}^{m} \times V$ of variational solutions of equation (5.1) which intersects $\{0\} \times V$, has dimension at each point at least $m$ and whose projection onto $\mathbf{R}^{m}$ is essential among proper maps.

ProOF. Proposition 5.2 guarantees that $A-B: \mathbf{R}^{m} \times V \rightarrow V^{*}$ is A-proper with respect to $\left\langle\left\{V_{j}\right\},\left\{P_{j}^{*}\left(V_{j}\right)\right\},\left\{P_{j}^{*}\right\}\right\rangle$. Let $\pi$ be the projection of $\mathbf{R}^{m} \times V$ onto $\mathbf{R}^{m}$. We claim that $\pi$ complements $A-B$ on all of $\mathbf{R}^{m} \times V$.

Indeed, assumption (5.6) implies that

$$
(A(v), v) \geq C_{0}\|v\|^{P}-C_{1} \quad \text { for all } v \in V .
$$

On the other hand, assumption (5.4) implies that when $\varepsilon>0$

$$
\|B(\lambda, v)\| \leq \frac{C_{0}}{2}\|v\|^{p-1}+C(\varepsilon) \quad \text { for all } v \in V
$$

where $C(\varepsilon)$ is independent of $v$.

If the assertion of (5.4) holds with $\varepsilon>0$, then

$$
(A(v)-B(\lambda, v), v) \geq \frac{C_{0}}{2}\|v\|^{p}-C(\varepsilon)\|v\|-C_{1} \quad \text { for all } v \in V
$$

so that we may choose $R>0$ such that $(A(v)-B(\lambda, v), v)>0$ when $\|v\| \geq R$. In particular, $\left(P_{k}^{*}(A(v)-B(\lambda, v)), v\right)>0$, when $\|v\| \geq R, v \in V_{k}$, and $k$ is any positive integer. Thus, the A-proper degree of $A-B(0, \cdot)$ is $\{1\}$ on all of $V$; i.e., $\pi$ complements $A-B$ on $\mathbf{R}^{m} \times V$.

If the second assertion of (5.10) holds, then

$$
(A(u)-B(\lambda, u), u) \geq C_{0}\|u\|^{p}-C_{1} \text { for all } u,
$$

and we also obtain the same conclusion.

To apply Theorem 1.2 it remains to verify that $\pi$ is proper on $\{(\lambda, v) \mid A(v)-$ $B(\lambda, v)=0\}$. But if $\left\{\left(\lambda_{k}, v_{k}\right)\right\}$ is such that $\left\{\lambda_{k}\right\}$ is bounded, and $A\left(v_{k}\right)-B\left(\lambda_{k}, u_{k}\right)$ $=0$ for all $k$, then from the above coerciveness conclusions it follows that $\left\{v_{k}\right\}$ is bounded. Then again using the reflexivity of $V$, Proposition 5.1, and the Rellich theorem, we can extract a subsequence of $\left\{\left(\lambda_{k}, v_{k}\right)\right\}$ which converges strongly to $(\lambda, v)$ with $A(v)-B(\lambda, v)=0$.

REMARK 5.1. By taking fuller advantage of the embedding theorems one can relax the growth conditions in (5.4) somewhat.

REMARK 5.2. Assumption (5.5) is a monotonicity assumption only on the highest-order derivatives. Under monotonicity assumptions involving all of the terms one can ensure that $A: V \rightarrow V^{*}$ is strongly monotone. In this case the assertion of Proposition 5.1 is immediate and our development is completely selfcontained.

REMARK 5.3. In the proof of Theorem 5.1 we invoked Theorem 1.2. One can also apply Theorem 1.2 to obtain conclusions on the solution set of (5.1) in $O \subseteq \mathbf{R}^{m} \times V$, where $O$ is open and $O \cap(\{0\} \times V) \supseteq\{0\} \times B(0, R)$, where $R$ is sufficiently large. 
The second problem we wish to consider is the following:

$$
\left\{\begin{array}{l}
\sum_{|\alpha| \leq 2 k} A_{\alpha}(x) D^{\alpha}(u)(x)=f\left(x, \xi_{2 k}(u)(x)\right), \quad x \in \Omega, \\
B_{i}(u)(x)=0, \quad x \in \partial \Omega, 1 \leq i \leq k-1 .
\end{array}\right.
$$

We assume that the coefficients $A_{\alpha}$ are smooth and such that

$$
L(u)(x)=\sum_{|\alpha| \leq 2 k} A_{\alpha}(x) D^{\alpha}(u)(x)
$$

defines a uniformly elliptic operator. Moreover, assume that the boundary operators $\left\{B_{i} \mid 1 \leq i \leq k-1\right\}$ cover $L$ in the sense of $[\mathbf{1}]$.

Under these assumptions there exists a closed subspace $V$ of $W^{2 k, 2}(\Omega)$ such that $L$ together with the boundary operators are realized by a bounded linear operator $\mathcal{L}: V \rightarrow L^{2}(\Omega)$. The ellipticity assumption guarantees that the null space of $\mathcal{L}, N(\mathcal{L})$, is of finite dimension, and that the range of $\mathcal{L}, R(\mathcal{L})$, is closed. The smoothness of the $A_{\alpha}$ 's guarantees that the codimension of $R(\mathcal{L})$ is also finite. Thus $\mathcal{L}: V \rightarrow L^{2}(\Omega)$ is Fredholm, and we will apply our previous results when

$$
\operatorname{ind}(\mathcal{L}) \equiv \operatorname{dim} N(\mathcal{L})-\operatorname{codim} R(\mathcal{L}) \equiv m>0 .
$$

A basic assumption of $f$ is that it has sublinear growth:

$$
\begin{aligned}
& f: \Omega \times \mathbf{R}^{s_{2 k}} \rightarrow \mathbf{R} \text { satisfies the Carathéodory conditions and there } \\
& \text { exist } a, b \in L^{2}(\Omega) \text { and } \sigma \in[0,1) \text { such that }|f(x, \xi)| \leq a(x)+b(x)|\xi|^{\sigma} \\
& \text { for } x \in \Omega, \xi \in \mathbf{R}^{s_{2 k}} \text {. }
\end{aligned}
$$

As in the first problem we allow the nonlinearity to depend on derivatives of order $2 k$. Whereas in the first problem we imposed a monotonicity condition on those variables of $f$ which interact with the highest-order derivatives, we here impose a Lipschitzian condition:

(5.16) if $\xi=(\eta, \nu)$ denotes the division of $\xi \in \mathbf{R}^{s_{2 k}}$ into its $2 k$ th order components, represented by $\nu$, and its lower-order components, represented by $\eta$, then there exists a $\beta \geq 0$ such that $\mid f(x, \eta, \nu)-$ $f(x, \eta, \bar{\nu})|\leq \beta| \nu-\bar{\nu} \mid$ for $x \in \Omega,(\eta, \nu)$ and $(\eta, \bar{\nu})$ in $\mathbf{R}^{s_{2 k}}$, and $\beta<\inf \left\{\|\mathcal{L}(v)\|_{L^{2}} \mid v \in(N(\mathcal{L}))^{\perp},\|v\|_{W^{2 k, 2}}=1\right\}$. $f:$

We finally impose the assumptions on the asymptotic interaction between $\mathcal{L}$ and

(5.17) letting $\sigma$ be as in (5.15) we assume that $f(x, \xi)=g(x, \xi)+h(x, \xi)$ for $x \in \Omega, \xi \in \mathbf{R}^{s_{2 k}}$, where

(i) $\lim _{s \rightarrow \pm \infty}\left(g(x, s, \eta) /|s|^{\sigma}\right)=g \pm(x)$, the limit being uniform in $\eta \in \mathbf{R}^{s_{2 k-1}}$

(ii) there exist $c, d \in L^{2}(\Omega)$ and $\varepsilon>0$ such that $|h(x, \xi)| \leq$ $c(x)+d(x)|\xi|^{\sigma-\varepsilon}$ for $x \in \Omega, \xi \in \mathbf{R}^{s_{2 k}}$;

there exists a subspace $Z$ of $N(\mathcal{L})$ and a linear bijection $T: Z \rightarrow(R(\mathcal{L}))^{\perp}$ such that
(i) $T(w)=0$ a.e. on $\{x \mid x \in \Omega, w(x)=0\}$, when $w \in Z$.
(ii) $\int_{w>0} g_{+}|w|^{\sigma} T(w) d x+\int_{w<0} g_{-}|w|^{\sigma} T(w) d x>0$ if $w \in Z-\{0\}$. 
THEOREM 5.2. Suppose that $\mathcal{L}$ and $f$ satisfy assumptions (5.14)-(5.18). Let $W$ denote the orthogonal complement in $N(\mathcal{L})$ of the subspace $Z$ of assumption (5.18). Then there exists a connected subset $C$ in $V$ of solutions of equation (5.13) whose dimension at each point is at least $m$, and such that the $V$-orthogonal projection of $V$ onto $W$ maps $C$ onto $W$.

ProOF. We begin by rewriting equation (5.13). Let $Q$ be the $L^{2}$-orthogonal projection of $L^{2}$ onto $R(\mathcal{L})$, and let $P$ be the $V$-orthogonal projection of $V$ onto $N(\mathcal{L})$. Let $S: N(I-Q) \rightarrow Z$ be any linear bijection. Then, clearly, if we let $K: R(\mathcal{L}) \rightarrow N(I-P)$ be the inverse of $L: N(I-P) \rightarrow R(\mathcal{L})$, it follows that $K \circ Q+S \circ(I-Q): L^{2} \rightarrow V$ is injective. Consequently, equation (5.13) is equivalent to

$$
H(u) \equiv[K \circ Q+S \circ(I-Q)][\mathcal{L}+F](u)=0, \quad u \in V,
$$

where $F$ is the Nemytsky operator generated by $f$.

Then, letting $W$ be the orthogonal complement in $N(\mathcal{L})$ of $Z$, it follows that $H: W \oplus W^{\perp} \rightarrow W^{\perp}$. We wish to show that $H$ is A-proper with respect to a suitable projection scheme.

Since $W^{\perp}$ is a separable Hilbert space we may choose an increasing sequence $\left\{X_{n}\right\}$ of finite-dimensional subspaces of $W^{\perp}$ with the property that if $P_{n}: W^{\perp} \rightarrow X_{n}$ is the $V$-orthogonal projection of $W^{\perp}$ onto $X_{n}$ for each $n$, then $\left\{P_{n}(u)\right\} \rightarrow u$ for each $u \in W^{\perp}$. Let $\Gamma$ be the approximation scheme for mappings of $W \oplus W^{\perp}$ into $W^{\perp}$ defined by $\Gamma=\left\langle\left\{W \oplus X_{n}\right\},\left\{X_{n}\right\},\left\{P_{n}\right\}\right\rangle$. We claim that $H$ is A-proper with respect to $\Gamma$.

Thus, let $V_{n}=W \oplus X_{n}$, and $T_{n}: V \rightarrow V_{n}$ be the $V$-orthogonal projection of $V$ onto $V_{n}$ for each $n$. Observe that $P_{n} H=T_{n} H$ for each $n$, so that $H: W \oplus W^{\perp} \rightarrow W^{\perp}$ is A-proper with respect to $\Gamma$ precisely when $H: V \rightarrow V$ is A-proper with respect to $\Gamma^{\prime} \equiv\left\langle\left\{V_{n}\right\},\left\{V_{n}\right\},\left\{T_{n}\right\}\right\rangle$.

Since $\Gamma^{\prime}$ is an approximation scheme which has the property that $\left\{T_{n}(h)\right\} \rightarrow h$ for each $h \in V$, it is easy to see that A-properness with respect to $\Gamma^{\prime}$ is a property which remains invariant under compact perturbations (see [19]). On the other hand,

$$
H(u)=u+K Q F(u)+[S \circ(I-Q)(\mathcal{L}+F)-P(u)] \text { for } u \in H,
$$

so that since $F$ maps bounded sets of $V$ to bounded sets of $L^{2}$ and $S$ and $P$ are both finite-dimensional operators, it will suffice to prove that $I+M: V \rightarrow V$ is A-proper with respect to $\Gamma^{\prime}$, where $M(u)=K Q F(u)$ for $u \in V$.

Using obvious notation we define $E: V \times V \rightarrow L^{2}$ by

$$
(E(v, u))(x)=f\left(x, \xi_{2 k-1}(v)(x), D^{2 k}(u)(x)\right)
$$

for $u, v \in V$ and $x \in \Omega$.

From the continuity and boundedness of $F: V \rightarrow L^{2}$ together with the Rellich compactness theorem it follows that if $u \in V$ and $\left\{v_{n}\right\} \subseteq V$ converges weakly to $v \in V$, then $\left\{E\left(v_{n}, u\right)\right\}$ converges strongly in $L^{2}$ to $E(v, u)$.

Now assumption (5.16) implies that $\left\|E\left(v, u_{1}\right)-E(v, u)\right\|_{L^{2}} \leq \beta\left\|u_{1}-u_{2}\right\|_{V}$. Thus, since $Q$ is an orthogonal projection it follows that

$$
\left\|K Q E\left(v, u_{1}\right)-K Q E\left(v, u_{2}\right)\right\|_{V} \leq \beta\|K\|\left\|v_{1}-v_{2}\right\|_{V} \quad \text { for } u_{1}, u_{2}, v \in V .
$$

We have shown that $K Q E: V \times V \rightarrow V$ is completely continuous with respect to its first variable and, since $\beta\|K\|<1$, a contraction with respect to its second variable. It follows that the mapping $M: V \rightarrow V$ is condensing with respect to the 
ball-measure of noncompactness (see Webb [24]). Consequently, since $\left\|T_{n}\right\| \leq 1$ for each $n, I+M: V \rightarrow V$ is A-proper with respect to $\Gamma^{\prime}$ (see Webb [23] and Nussbaum [17]). Thus $H: W \oplus W^{\perp} \rightarrow W^{\perp}$ is A-proper with respect to $\Gamma$.

We now claim that if $\hat{P}$ denotes the orthogonal projection of $V$ onto $W$, then $\hat{P}$ is a complement for $H$ on all of $V$. To verify this claim it suffices, by Proposition 3.1 , to prove that $\operatorname{deg}\left(\left.H\right|_{W^{\perp}}, W^{\perp}, 0\right) \neq\{0\}$.

We define a homotopy $\Phi:[0,1] \times W^{\perp} \rightarrow W^{\perp}$ by

$$
\Phi(t, u)=[K \circ Q+S \circ(I-Q)][\mathcal{L}(u)+t T \circ P(u)+(1-t) F(u)] .
$$

By the same argument used above, $\Phi(t, \cdot): W^{\perp} \rightarrow W^{\perp}$ is A-proper for each $t \in[0,1]$, it is continuous, and the continuity is uniform on bounded subsets of $[0,1] \times W^{\perp}$. Thus it follows from the homotopy invariance of the A-proper degree (see $[\mathbf{8}]$ ) that if we can find an a priori bound for solutions of

$$
\Phi(t, u)=0, \quad 0 \leq t \leq 1, u \in W^{\perp},
$$

it will follow that $\operatorname{deg}\left(\Phi(0, \cdot), W^{\perp}, 0\right)=\operatorname{deg}\left(\Phi(1, \cdot), W^{\perp}, 0\right)$,

or

$$
\operatorname{deg}\left(H, W^{\perp}, 0\right)=\operatorname{deg}\left(\Phi(0, \cdot), W^{\perp}, 0\right) .
$$

Since $\Phi(0, \cdot)$ is linear and invertible, $\operatorname{deg}\left(\Phi(0, \cdot), W^{\perp}, 0\right) \neq\{0\}$.

To obtain the a priori bound for solutions for equation (5.20), we assume the contrary. Suppose that there exist $\left\{u_{n}\right\} \subseteq W^{\perp}$ and $\left\{t_{n}\right\} \subseteq[0,1]$ with $\left\{\left\|u_{n}\right\|\right\} \rightarrow$ $+\infty$ and $\left(t_{n}, u_{n}\right)$ a solution of equation (5.20) for each $n$. (Here $\|u\|$ denotes the norm in $W^{2 k}$.)

Hence,

$$
\mathcal{L}\left(u_{n}\right)+t T \circ P\left(u_{n}\right)+(1-t) F\left(u_{n}\right)=0 \text { for each } n \text {. }
$$

We write $u_{n}=w_{n}+z_{n}$, where $w_{n} \in N(P)$ and $z_{n} \in Z$, for each $n$.

By composing equation (5.21) with $Q$ we see that $\mathcal{L}\left(w_{n}\right)+\left(1-t_{n}\right) Q F\left(u_{n}\right)=0$ for each $n$, and since

$$
\lim _{n \rightarrow \infty} \frac{\left\|F\left(u_{n}\right)\right\|_{L^{2}}}{\left\|u_{n}\right\|_{V}}=0
$$

and there is a constant $c>0$ such that $\left\|w_{n}\right\|_{V} \leq c\left\|\mathcal{L}\left(w_{n}\right)\right\|_{L^{2}}$ for each $n$, it follows that $\left\{w_{n} /\left\|u_{n}\right\|\right\} \rightarrow 0$. Since $Z$ is finite dimensional, we may suppose that $\left\{z_{n} /\left\|u_{n}\right\|\right\} \rightarrow z^{*} \in Z$. Hence $\left\{u_{n} /\left\|u_{n}\right\|\right\} \rightarrow z^{*}$ and $z^{*} \neq 0$. Also, we may assume that $\left\{u_{n} /\left\|u_{n}\right\|\right\}$ converges pointwise a.e. to $z^{*}$ on $\Omega$.

Now for each $x \in \Omega$ and $u \in \mathbf{N}$ with $u_{n}(x) \neq 0$, we have

$$
\frac{F\left(u_{n}\right)(x)}{\left\|u_{n}\right\|^{\sigma}}=\frac{g\left(x, u_{n}(x), \xi_{2 k}\left(u_{n}\right)(x)\right)}{\mid u_{n}(x)^{\sigma}}\left|\frac{u_{n}(x)}{\left\|u_{n}\right\|}\right|^{\sigma}+\frac{h\left(x, \xi_{2 k}\left(u_{n}\right)(x)\right)}{\left\|u_{n}\right\|^{\sigma}} .
$$

From (5.17) we see that without loss of generality we may assume that

$$
\left\{\frac{h\left(x, \xi_{2 k}\left(u_{n}\right)(x)\right)}{\left\|u_{n}\right\|^{\sigma}}\right\} \rightarrow 0 \text { a.e. on } \Omega
$$

and also that $F\left(u_{n}\right)(x) /\left\|u_{n}\right\|^{\sigma} \rightarrow I\left(z^{*}\right)(x)$ a.e. on $\left\{x \mid z^{*}(x) \neq 0\right\}$, where $I\left(z^{*}\right)(x)=$ $g_{+}(x)\left|z^{*}(x)\right|^{\sigma}$ if $z^{*}(x)>0$ and $I\left(z^{*}\right)(x)=g_{-}(x)\left|z^{*}(x)\right|^{\sigma}$ when $z^{*}(x)<0$. Moreover, from assumption (5.15) we may, without loss of generality, suppose that $\left\{F\left(u_{n}\right) /\left\|u_{n}\right\|^{\sigma}\right\}$ converges weakly in $L^{2}(\Omega)$ to $w^{*}$. Then, clearly, $w^{*}=I\left(z^{*}\right)$ a.e. on $\left\{x \mid z^{*}(x) \neq 0\right\}$. 
Using (5.18)(i) it follows that

$$
\lim _{n \rightarrow \infty} \int_{\Omega} \frac{F\left(u_{n}\right)(x)}{\left\|u_{n}\right\|^{\sigma}} T\left(z^{*}\right)(x) d x=\int_{\Omega} I\left(z^{*}\right)(x) T\left(z^{*}\right)(x) d x,
$$

and by (5.18)(ii) this limit is positive.

Finally, for each $n$, compose equation (5.21) with $(I-Q)$ and take the $L^{2}$-linear product with $T\left(z^{*}\right)$. Then

$$
\begin{aligned}
& t_{n}\left\|u_{n}\right\| \int_{\Omega} T \frac{\left(z_{n}\right)(x)}{\left\|u_{n}\right\|} T\left(z^{*}\right)(x) d x \\
& \quad+\left(1-t_{n}\right)\left\|u_{n}\right\|^{\sigma} \int_{\Omega} \frac{F\left(u_{n}\right)(x)}{\left\|u_{n}\right\|^{\sigma}} T\left(z^{*}\right)(x) d x=0 \text { for each } n .
\end{aligned}
$$

Since the sequence $\left\{\int_{\Omega}\left(T\left(z_{n}\right)(x) /\left\|u_{n}\right\|\right) T\left(z^{*}\right)(x) d x\right\}$ converges to $\left\|T\left(z^{*}\right)\right\|_{L^{2}}^{2}>$ 0 , we clearly have a contradiction.

Thus the a priori bound holds and $\hat{P}$ complements $H$ on all of $V$.

To apply Theorem 1.2 it remains to verify the properness assumption of the complementing map on the solution set.

This is equivalent to proving that if $\left\{v_{n}\right\} \subseteq V$ is such that $\mathcal{L}\left(u_{n}\right)+F\left(u_{n}\right)=0$ for each $n$, and $\left\{\hat{P}\left(v_{n}\right)\right\}$ is bounded, then $\left\{v_{n}\right\}$ has a convergent sequence. But an argument almost identical with the last completion argument guarantees that such a sequence $\left\{v_{n}\right\}$ must be bounded in $V$. Since $H$ is a condensing perturbation of the identity, its restriction to closed bounded sets is proper. It follows that a subsequence of $\left\{v_{n}\right\}$ converges to $V$ and $\mathcal{L}(u)+F(u)=0$.

We can now apply the general version Theorem 1.2 to obtain our desired conclusion.

REMARK 5.4. In the case when $\sigma=0$, the nonlinearity does not depend on derivatives of $u$, and $\lim _{s \rightarrow \pm \infty} f(x, s)=f \pm(x)$, the limit being uniform in $x \in \Omega$ (the conclusion of Theorem 5.2 was obtained in [16]). Under the same hypotheses as in [3], the existence of a set of solutions of equation (5.13) whose projection onto $W$ covers $W$ was proven when $\operatorname{dim}(W)=1$, and for general $W$ when $f$ is assumed to be Lipschitz with respect to $u$. No connectedness or dimension assertions were considered in the latter. In [18] conclusions are obtained for equations like (5.13) which are similar to those of Theorem 5.2, but without assertions concerning the dimension of $C$ and under different asymptotic conditions.

REMARK 5.5. In the case when $\mathcal{L}: V \rightarrow L^{2}$ is Fredholm of index 0 , the proof we have used for Theorem 5.2 yields the existence of at least one solution of $\mathcal{L}(u)+$ $F(u)=0$; instead of quoting Theorem 1.2 one just uses the existence property of topological degree. The existence result so obtained strengthens the existence results of [13] and of [21], in that the condition corresponding to (5.18) is stronger in both these papers (for instance, in these papers $\mathcal{L}$ is assumed to satisfy a unique continuation condition), and the growth condition corresponding to (5.17) is more restrictive in $[\mathbf{2 1}]$. The proof that we give above is considerably simpler than those of $[\mathbf{1 3}$ and $\mathbf{2 1}]$.

REMARK 5.6. Inequalities of the type (5.18)(ii) are an outgrowth of the Landesman-Lazer conditions when $(R(\mathcal{L}))^{\perp}=N(\mathcal{L}), T=I$, and when $\sigma=0$. In case $(R(\mathcal{L}))^{\perp} \neq N(\mathcal{L}), \sigma=0$, and $\operatorname{index}(\mathcal{L})=0$, inequalities of type (5.18)(ii) were first introduced in $[\mathbf{2 1}]$, in conjunction with a more restrictive vanishing condition than 
(5.18)(i). Observe that (5.18)(i) is always satisfied when $\mathcal{L}$ has either the unique continuation property or when $(R(\mathcal{L}))^{\perp} \subseteq N(\mathcal{L})$ and one takes $T=I$.

\section{REFERENCES}

1. S. Agmon, A. Douglis and L. Nirenberg, Estimates near the boundary of elliptic partial differential equations satisfying general boundary conditions. I, Comm. Pure Appl. Math. 12 (1959), 623-727.

2. J. C. Alexander and J. A. Yorke, The implicit function theorem and the global methods of cohomology, J. Funct. Anal. 21 (1976), 330-339.

3. H. Amann, A. Ambrosetti and G. Mancini, Elliptic equations with noninvertible Fredholm linear part and bounded nonlinearities, Math. Z. 158 (1979), 179-194.

4. G. E. Bredon, Equivariant cohomology theories, Lecture Notes in Math., vol. 34, SpringerVerlag, Berlin and New York, 1967.

5. F. E. Browder, On continuity of fixed points under deformations of continuous mappings, Summa Brasil Mat. 4 (1960), 183-190.

6. __ Existence theorems for nonlinear partial differential equations, Global Analysis, Proc. Sympos. Pure Math., vol. 16, Amer. Math. Soc., Providence, R.I., 1970.

7. __ Nonlinear operators and nonlinear equations of evolution in Banach spaces, Proc. Sympos. Pure Math., vol. 18, Part 2, Amer. Math. Soc., Providence, R.I., 1976.

8. F. E. Browder and W. V. Petryshyn, Approximation methods and the generalized topological degree for nonlinear mappings in Banach spaces, J. Funct. Anal. 3 (1969), 385-414.

9. A Dold, Lectures on algebraic topology, Springer-Verlag, Berlin and New York, 1972.

10. R. Engleking, Dimension theory, North-Holland, Amsterdam, 1978.

11. P. M. Fitzpatrick, I. Massabó and J. Pejsachowicz, Multiple solutions for nonlinear Fredholm operators (in preparation).

12. M. Furi and M. P. Pera, On the existence of an unbounded connected set of solutions for nonlinear equations in Banach spaces, Accad. Naz. Lincei (7) 67 (1979), 31-38.

13. G. Hetzer, $A$ note on a paper of Howard Shaw concerning a nonlinear elliptic boundary value problem, J. Differential Equations 32 (1979), 186-192.

14. J. Leray and J.-P. Lions, Quelques résultats de Visik sur les problèmes elliptiques nonlinéaires par les méthodes de Minty-Browder, Bull. Soc. Math. France 93 (1965), 97-107.

15. J. Leray and I. Schauder, Topologie et équations fonctionnelles, Ann. Sci. Ėcole Norm. Sup. 51 (1934), 45-78.

16. I. Massabó and J. Pejsachowicz, On the connectivity properties of the solution set of parametrized families of compact vector fields, J. Funct. Anal. 59 (1984), 151-166.

17. R. D. Nussbaum, Degree theory for local condensing maps, J. Math. Anal. Appl. 37 (1972), 741-766.

18. M. P. Pera, Unbounded components of solutions of nonlinear equations at resonance, and an application to elliptic boundary value problems (to appear).

19. W. V. Petryshyn, The approximation solvability of equations involving A-proper and pseudo-A-proper mappings, Bull. Amer. Math. Soc. 81 (1975), 223-312.

20. P. H. Rabinowitz, $A$ note on a nonlinear elliptic equation, Indiana Univ. Math. J. 22 (1972), 43-49.

21. H. Shaw, A nonlinear eigenvalue problem at resonance, J. Differential Equations 26 (1977), 335-346.

22. E. Spanier, Algebraic topology, McGraw-Hill, New York, 1966.

23. J. R. L. Webb, Mapping and fixed-point theorems for nonlinear operators in Banach spaces, Proc. London Math. Soc. 20 (1970), 451-468.

24. __ Remarks on k-set contractions, Boll. Un. Mat. Ital. 4 (1971), 775-788.

Department of Mathematics, University of MARYland, College Park, MaryLAND 20742 (Current address of P. Fitzpatrick)

Dipartimento di Matematica, Universitá della Calabria, Cosenza, Italy (Current address of I. Massabó and J. Pejsachowicz) 